\title{
Amplification of Asymmetric Induction in Sequential Reactions of Bis-diazoacetates Catalyzed by Chiral Dirhodium(II) Carboxamidates
}

\author{
Michael P. Doyle,* Yuanhua Wang, Pejman Ghorbani and Erhard Bappert \\ Department of Chemistry and Biochemistry, University of Maryland, College Park, Maryland \\ 20742
}

\section{Supporting Information}

General. NMR spectra were recorded on Bruker DRX-250 MHz, Bruker DRX-500 MHz or DRX-600 MHz instruments. Chemical shifts are reported in parts per million (ppm, $\delta$ ) downfield from the internal standard, $\mathrm{Me}_{4} \mathrm{Si}$ (TMS) for ${ }^{1} \mathrm{H}$ NMR spectra. ${ }^{13} \mathrm{C}$ NMR data is reported relative to the central line of the chloroform signal $(\delta=77.00 \mathrm{ppm})$. Highresolution mass spectra were obtained on a JEOL HX110A spectrometer. Low-resolution mass spectra were obtained on a Finnigan LCQMS spectrometer. Infrared spectra were recorded as indicated; absorption is reported in wave-numbers $\left(\mathrm{cm}^{-1}\right)$. Thin layer chromatography was performed on Merck silica gel $60 \quad \mathrm{~F}_{254}$ glass backed plates; visualization was achieved with UV, iodine, or phosphomolybdic acid. Column chromatography was performed on silica gel (230-400 mesh). Dichloromethane was purified by pressure filtration through activated alumina. Tetrahydrofuran was distilled from sodium benzophenone ketyl, and other solvents were used as their commercial anhydrous grade. 


\section{Synthesis of trans-Cylohexane-1,4-diyl Bis-diazoacetate (1).}

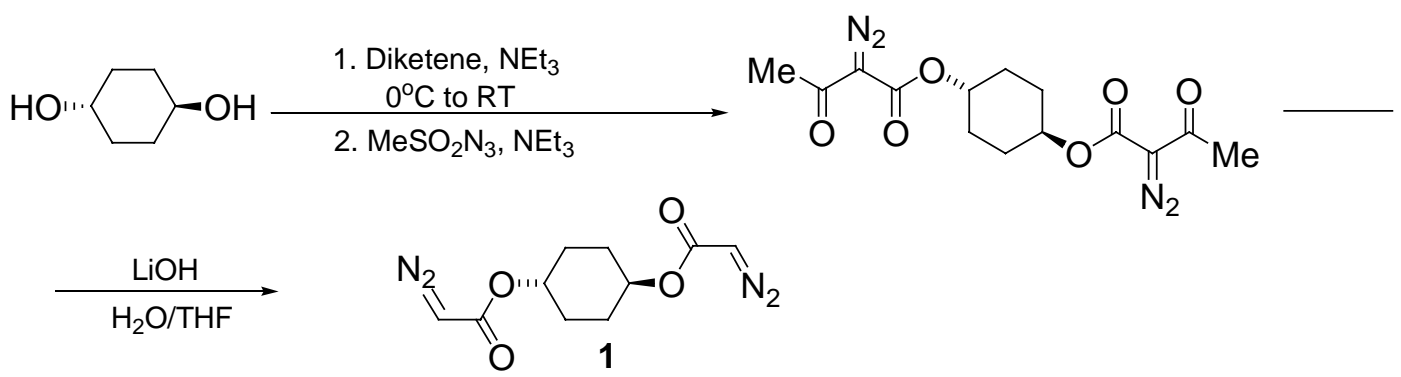

trans-Cylohexane-1,4-diol. Commercially available cylohexane-1,4-diol (26.0 g, 224 $\mathrm{mmol}$ ), with a cis/trans ratio of 50/50 was placed in $300 \mathrm{~mL}$ of acetone and heated at reflux. The solution was cooled to $0^{\circ} \mathrm{C}$ over night. White needles were formed, which were filtered and washed with cold acetone. This process was repeated one more time to yield $3.49 \mathrm{~g}$ of pure trans-cylohexane-1,4-diol as white needles: $\mathrm{mp} 143-144^{\circ} \mathrm{C}$ (lit. ${ }^{1} \mathrm{mp}$ $\left.143^{\circ} \mathrm{C}\right)$.

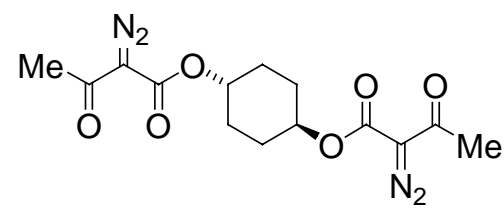

trans-Cylohexane-1,4-diyl Bis-(2'-diazo-3'-oxobutyrate). To a solution of transcylohexane-1,4-diol (2.94 g, $25.3 \mathrm{mmol})$ in $100 \mathrm{~mL}$ dry THF was cooled to $0^{\circ} \mathrm{C}$ was added triethylamine $(0.71 \mathrm{~mL}, 5.1 \mathrm{mmol})$ and diketene $(6.29 \mathrm{~g}, 76.0 \mathrm{mmol})$. The reaction mixture was allowed to warm to room temperature. After $24 \mathrm{~h}$, the solution was again cooled to $0^{\circ} \mathrm{C}$, at which time triethylamine $(10.29 \mathrm{~mL}, 76.0 \mathrm{mmol})$ and methanesulfonyl azide $(9.17 \mathrm{~g}, 76.0 \mathrm{mmol})$ were added. The dark brown solution was allowed to warm slowly to room temperature and was stirred for additional $24 \mathrm{~h}$. The reaction mixture was separated by flash chromatography on silica gel (elution with 1:3 ethyl acetate:hexanes), and $5.53 \mathrm{~g} \mathrm{(65 \%} \mathrm{yield)} \mathrm{of} \mathrm{the} \mathrm{title} \mathrm{compound} \mathrm{was} \mathrm{crystallized} \mathrm{from} \mathrm{ethyl} \mathrm{acetate/hexanes}$ (9/1) as yellow plates: $\mathrm{mp} 147^{\circ} \mathrm{C}$ (decomposition); IR (KBr): $2148\left(\mathrm{C}=\mathrm{N}_{2}\right), 1709(\mathrm{C}=\mathrm{O})$

1. Kikuchi, Y.; Kato, Y.; Tanaka, Y.; Toi, H.; Aoyama, Y. J. Am. Chem. Soc. 1991, 113, 1349. 
and $1654(\mathrm{C}=\mathrm{O}) \mathrm{cm}^{-1} ;{ }^{1} \mathrm{H}$ NMR $\left(500 \mathrm{MHz}, \mathrm{CDCl}_{3}\right): \delta$ 5.03-4.95 (comp, 2H), $2.48(\mathrm{~s}, 6 \mathrm{H})$, 2.07-2.01 (comp, 4H), 1.73-1.64 (comp, 4H); ${ }^{13} \mathrm{C}$ NMR (62.5 MHz, $\mathrm{CDCl}_{3}$ ): $\delta 189.9$, 160.8, 71.8, 28.2, 27.8; HRMS-FAB $(m / z):[\mathrm{M}+\mathrm{H}]^{+}$calcd for $\mathrm{C}_{14} \mathrm{H}_{17} \mathrm{~N}_{4} \mathrm{O}_{6}, 337.1148$; found, 337.1143.

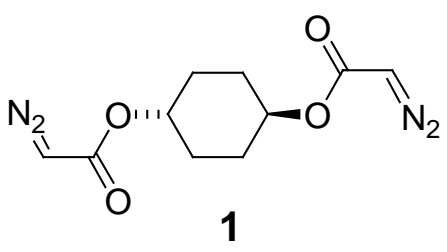

trans-Cylohexane-1,4-diyl Bis-diazoacetate(1). A solution of LiOH monohydrate (266 $\mathrm{mg}, 6.34 \mathrm{mmol}$ ) in $20 \mathrm{~mL}$ distilled water was added to a solution of trans-cylohexane1,4-diyl bis-(2'-diazo-3'-oxobutyrate) $(710 \mathrm{mg}, 2.11 \mathrm{mmol})$ in $20 \mathrm{~mL}$ of dry THF. The progress of the reaction was monitored by ${ }^{1} \mathrm{H}-\mathrm{NMR}$. After completion of the cleavage reaction, the reaction mixture was diluted with $50 \mathrm{~mL}$ water and $100 \mathrm{~mL}$ ethyl acetate. The organic layer was separated, and the aqueous layer was extracted with ethyl acetate $(2 \times 100 \mathrm{~mL})$. The combined organic layer was dried over anhydrous $\mathrm{MgSO}_{4}$, and the solvent was removed under reduced pressure. Column chromatography on silica gel (elution with 1:3 ethyl acetate:pentane) provided $480 \mathrm{mg}(90 \%)$ of the diazo compound 1 as yellow crystals: mp 124-126 ${ }^{\circ} \mathrm{C}$; IR $(\mathrm{KBr}): 2110\left(\mathrm{C}=\mathrm{N}_{2}\right)$ and $1666(\mathrm{C}=\mathrm{O}) \mathrm{cm}^{-1}$; ${ }^{1} \mathrm{H}$ NMR (500 MHz, $\mathrm{CDCl}_{3}$ ): $\delta$ 4.95-4.82 (comp, 2H), 4.72 (br s, 2H), 2.05-1.89 (comp, 4H), 1.62-1.48 (comp, 4H); ${ }^{13} \mathrm{C}$ NMR $\left(62.5 \mathrm{MHz}, \mathrm{CDCl}_{3}\right): \delta 166.3,71.3,46.3,28.0$; HRMSFAB $(m / z):[\mathrm{M}+\mathrm{H}]^{+}$calcd for $\mathrm{C}_{10} \mathrm{H}_{13} \mathrm{~N}_{4} \mathrm{O}_{4}, 253.0937$; found, 253.0934 .

\section{General Procedure for Dirhodium(II) Catalyzed Decomposition of trans-} Cylohexane-1,4-diyl Bis-diazoacetate (1). Two procedures were used. Procedure A: The dirhodium(II) catalyst $(1.0 \mathrm{~mol} \%, 0.0040 \mathrm{mmol})$ was placed in an oven-dried, oneneck round bottom flask, equipped with a reflux condenser and a rubber septum and set under nitrogen. Then $10 \mathrm{~mL}$ of freshly distilled $\mathrm{CH}_{2} \mathrm{Cl}_{2}$ was added to dissolve the catalyst, and the resultant solution was stirred at reflux for $10 \mathrm{~min}$. trans-Cylohexane1,4-diyl bis-diazoacetate $(100 \mathrm{mg}, 0.40 \mathrm{mmol})$, dissolved in $10 \mathrm{~mL}$ of freshly distilled 
$\mathrm{CH}_{2} \mathrm{Cl}_{2}$, was added over $10 \mathrm{~h}$ via syringe pump $(1.0 \mathrm{~mL} / \mathrm{h})$. Upon completion of the addition, the solution was heated at reflux for an additional $2 \mathrm{~h}$; then solvent was removed under reduced pressure. The product ratio was monitored by ${ }^{1} \mathrm{H}-\mathrm{NMR}$ of the crude reaction mixture. Compounds $\mathbf{2}, \mathbf{3}$ and $\mathbf{4}$ were isolated by flash chromatography on silica gel (elution with 3:10 ethyl acetate:hexanes). Procedure B: The dirhodium(II) catalyst $(2.0 \mathrm{~mol} \%, 0.0040 \mathrm{mmol})$ was placed in an oven-dried, one-neck round bottom flask, equipped with a reflux condenser and a rubber septum and set under nitrogen. Then $10 \mathrm{~mL}$ of freshly distilled $\mathrm{CH}_{2} \mathrm{Cl}_{2}$ was added to dissolve the catalyst, and the resultant solution was stirred at reflux for $10 \mathrm{~min}$. trans-Cylohexane-1,4-diyl bisdiazoacetate $\left(50 \mathrm{mg}, 0.20 \mathrm{mmol}\right.$ ), dissolved in $5 \mathrm{~mL}$ of freshly distilled $\mathrm{CH}_{2} \mathrm{Cl}_{2}$, was added over $2 \mathrm{~h}$ via syringe pump $(2.5 \mathrm{~mL} / \mathrm{h})$. Upon completion of the addition, the solution was heated at reflux for an additional $10 \mathrm{~h}$; then solvent was removed under reduced pressure. The product ratio was monitored by ${ }^{1} \mathrm{H}-\mathrm{NMR}$ of the crude reaction mixture. Compounds $\mathbf{2}, \mathbf{3}$ and $\mathbf{4}$ were isolated by flash chromatography on silica gel (elution with 3:10 ethyl acetate:hexanes).

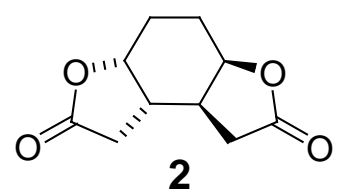

Octahydrobenzo[1,2-b,4,3-b' $]$ difuran-2,7-dione (2). Mp 128-129 ${ }^{\circ} \mathrm{C}$; IR (KBr): 1777 $(\mathrm{C}=\mathrm{O}) \mathrm{cm}^{-1} ;{ }^{1} \mathrm{H} \mathrm{NMR}\left(500 \mathrm{MHz}, \mathrm{CDCl}_{3}\right): \delta 4.68-4.62$ (comp, $2 \mathrm{H}$ ), 2.81 (dddd, $J=17.4$, 9.5, 7.2, 7.2 Hz, 2H), 2.31 (d, $J=17.2 \mathrm{~Hz}, 2 \mathrm{H}$ ), 2.31-2.24 (comp, 2H), 2.11-2.01 (comp, 2H), 1.88-1.77 (comp, 2H); ${ }^{13} \mathrm{C} \mathrm{NMR} \mathrm{(125} \mathrm{MHz,} \mathrm{CDCl}_{3}$ ): $\delta$ 175.5, 77.4, 35.9, 35.9, 21.1; HRMS-FAB $(m / z):[\mathrm{M}+\mathrm{H}]^{+}$calcd for $\mathrm{C}_{10} \mathrm{H}_{13} \mathrm{O}_{4}, 197.0814$; found, 197.0809. A single crystal of $\mathbf{2}$ was grown from a saturated solution in hexanes and ethyl acetate solution. 


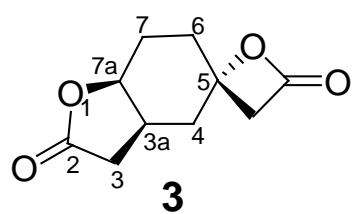

(3aS,5S,7aS)-5-(5-Hydroxy-hexahydrobenzofuran-2-one-5-yl)acetic Acid Lactone (3). Mp 94-95 ${ }^{\circ} \mathrm{C}$ (decomposition); $[\alpha]_{\mathrm{D}}^{27}=-34.8\left(c=0.62, \mathrm{CHCl}_{3}\right)$ with a sample obtained from the decomposition of 1 with $\mathrm{Rh}_{2}(4 S \text {-MPPIM })_{4}$; IR $(\mathrm{KBr}): 1819(\mathrm{C}=\mathrm{O})$ and 1774 $(\mathrm{C}=\mathrm{O}) \mathrm{cm}^{-1} ;{ }^{1} \mathrm{H}$ NMR $\left(500 \mathrm{MHz}, \mathrm{CDCl}_{3}\right): \delta 4.59-4.53(\mathrm{~m}, 1 \mathrm{H}), 3.23(\mathrm{~d}, J=16.3 \mathrm{~Hz}, 1 \mathrm{H})$, $3.18(\mathrm{~d}, J=16.3 \mathrm{~Hz}, 1 \mathrm{H}), 2.79$ (dd, $J=16.8,6.6 \mathrm{~Hz}, 1 \mathrm{H}), 2.75-2.18$ (m, 1H), 2.34-2.29 (m, 1H), 2.28 (d, $J=17.0 \mathrm{~Hz}, 1 \mathrm{H}), 2.10-1.94$ (comp, 3H), 1.89-1.84 (m, 1H), 1.61 (dd, $J$ $=13.6,12.6 \mathrm{~Hz}, 1 \mathrm{H}) ;{ }^{13} \mathrm{C} \mathrm{NMR}\left(62.5 \mathrm{MHz}, \mathrm{CDCl}_{3}\right): \delta 176.2,167.0,76.2,48.1,38.1$, 35.8, 32.8, 28.5, 23.3; LRMS-FAB $(\mathrm{m} / \mathrm{z}): 197[\mathrm{M}+\mathrm{H}]^{+}$. A single crystal 3 was grown from a saturated solution in hexanes and ethyl acetate. The enantiomeric excess of $\mathbf{3}$ was determined with baseline separation by GC on a 30-m Chiraldex B-DM column: $\mathrm{t}_{\mathrm{R}} 53.5$ $\min ((3 \mathrm{a} R, 5 R, 7 \mathrm{a} R)-\mathbf{3})$ and $55.9 \mathrm{~min}((3 \mathrm{a} S, 5 S, 7 \mathrm{a} S)-\mathbf{3})$. The enantiomeric excess of $\mathbf{3}$ was also determined by GC on a 30-m Chiraldex GTA column: $\mathrm{t}_{\mathrm{R}} 32.9$ min $((3 \mathrm{a} R, 5 R$, $7 \mathrm{a} R)-3)$ and $34.1 \mathrm{~min}((3 \mathrm{a} S, 5 S, 7 \mathrm{a} S)-3)$.

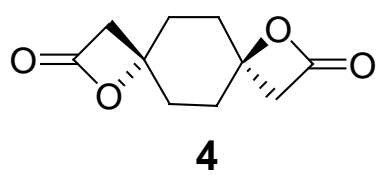

1,8-Dioxa-dispiro[3.2.3.2]dodecane-2,9-dione (4). $\mathrm{Mp} 99^{\circ} \mathrm{C}$ (decomposition); $\mathrm{IR}(\mathrm{KBr})$ : $1845(\mathrm{C}=\mathrm{O}) \mathrm{cm}^{-1} ;{ }^{1} \mathrm{H}$ NMR $\left(500 \mathrm{MHz}, \mathrm{CDCl}_{3}\right): \delta 3.21$ (s, 4H), 2.20-2.08 (comp, 4H), 2.07-1.97 (comp, 4H); ${ }^{13} \mathrm{C}$ NMR (125 MHz, $\mathrm{CDCl}_{3}$ ): $\delta 167.3$, 76.4, 47.4, 31.6. A single crystal of $\mathbf{4}$ was grown from a saturated solution in hexanes and ethyl acetate. 
Synthesis of E,E-2,4-Hexadien-1,6-diyl Bis-diazoacetate (5).
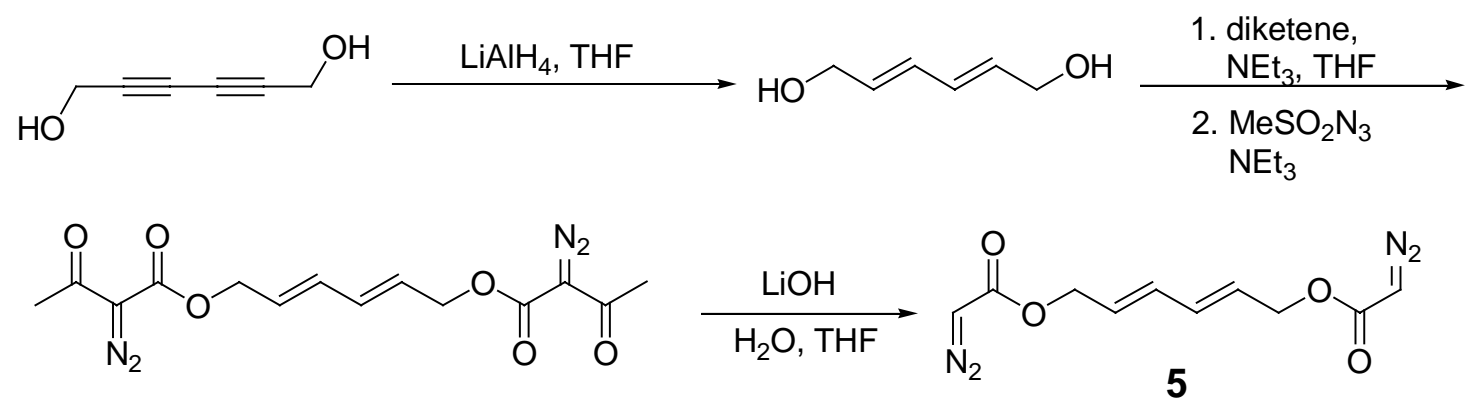

$\boldsymbol{E}, \boldsymbol{E}$-2,4-Hexadien-1,6-diol. A solution of 1,6-hexadiyne (5.23 g, $47.5 \mathrm{mmol})$ in $500 \mathrm{~mL}$ dry THF was cooled to $0^{\circ} \mathrm{C}$, and $\mathrm{LiAlH}_{4}(9.03 \mathrm{~g}, 238 \mathrm{mmol})$ was added in one-gram portions. After the grey suspension stopped bubbling, the reaction mixture was heated to reflux for $22 \mathrm{~h}$. The cooled suspension was diluted with $500 \mathrm{mLEt}_{2} \mathrm{O}$. To the resulting suspension was added $9 \mathrm{~mL}$ of $\mathrm{H}_{2} \mathrm{O}, 9 \mathrm{~mL}$ of $15 \%$ aqueous sodium hydroxide solution and, finally, $27 \mathrm{~mL} \mathrm{H}_{2} \mathrm{O}$. The cloudy solution was filtered, and the filtered material was rinsed with ethyl acetate. After drying the solution over anhydrous $\mathrm{MgSO}_{4}$ and evaporation of the solvent under reduced pressure, $3.90 \mathrm{~g}$ (72\% yield) of E,E-2,4hexadiene-1,6-diol was obtained as yellow needles: $\mathrm{mp} 103-105^{\circ} \mathrm{C}$ (lit. ${ }^{2} \mathrm{mp} 105.5$ $\left.106.5^{\circ} \mathrm{C}\right)$.<smiles>CC(=O)C(=N)C(=O)OC/C=C/C=C/COC(=O)C(=[W])C(C)=O</smiles>

$\boldsymbol{E}, \boldsymbol{E}$-2,4-Hexadien-1,6-diyl Bis-(2'-diazo-3'-oxobutyrate). To a solution of E,E-2,4hexadien-1,6-diol $(114 \mathrm{mg}, 1.00 \mathrm{mmol})$ in $5 \mathrm{~mL}$ dry $\mathrm{THF}$ was cooled to $0^{\circ} \mathrm{C}$ was added triethylamine (20 mg, $0.20 \mathrm{mmol})$ and diketene $(252 \mathrm{mg}, 3.00 \mathrm{mmol})$. After addition the reaction mixture was allowed to warm to room temperature. After $20 \mathrm{~h}$, the solution was again cooled to $0^{\circ} \mathrm{C}$, at which time triethylamine $(0.41 \mathrm{~mL}, 3.00 \mathrm{mmol})$ and methanesulfonyl azide (363 mg, $3.00 \mathrm{mmol}$ ) were added. The dark solution was allowed to warm slowly to room temperature and was stirred for additional $20 \mathrm{~h}$. Celite (1.00 g) 
was added, and the solvent was evaporated. Column chromatography on silica gel (elution with 1:3 ethyl acetate:hexanes) provided $120 \mathrm{mg}$ (36\% yield) of the diazo compound as a yellow solid: $\mathrm{mp} 112-114^{\circ} \mathrm{C}$ (decomposition); IR (KBr): $2148\left(\mathrm{C}=\mathrm{N}_{2}\right)$, $1709(\mathrm{C}=\mathrm{O})$ and $1654(\mathrm{C}=\mathrm{O}) \mathrm{cm}^{-1} ;{ }^{1} \mathrm{H}$ NMR $\left(250 \mathrm{MHz}, \mathrm{CDCl}_{3}\right): \delta 6.32(\mathrm{ddd}, J=14.6$, 9.1, $2.9 \mathrm{~Hz}, 2 \mathrm{H}), 5.90-5.78(\mathrm{comp}, 2 \mathrm{H}), 4.78(\mathrm{~d}, J=6.0 \mathrm{~Hz}, 2 \mathrm{H}), 2.49$ (s, 6H); ${ }^{13} \mathrm{C} \mathrm{NMR}$ $\left(62.5 \mathrm{MHz}, \mathrm{CDCl}_{3}\right): \delta 189.9,161.1,133.4,127.8,65.0,28.2 ; \operatorname{HRMS}-\mathrm{FAB}(\mathrm{m} / \mathrm{z}):[\mathrm{M}+$ $\mathrm{H}]^{+}$calcd for $\mathrm{C}_{14} \mathrm{H}_{15} \mathrm{~N}_{4} \mathrm{O}_{6}, 335.0992$; found, 335.0993.

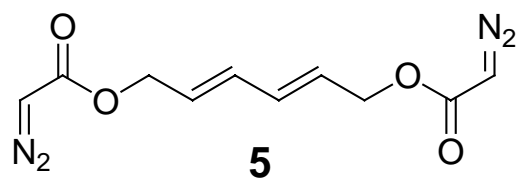

E,E-2,4-Hexadien-1,6-diyl Bis-diazoacetate (5). A solution of LiOH monohydrate (366 $\mathrm{mg}, 8.71 \mathrm{mmol}$ ) in $30 \mathrm{~mL}$ distilled $\mathrm{H}_{2} \mathrm{O}$ was added to a solution of E,E-2,4-hexadien-1,6diyl bis( $2^{\prime}$-diazo-3'-oxobutyrate) (970 mg, $\left.2.90 \mathrm{mmol}\right)$ in $30 \mathrm{~mL}$ of THF. The progress of the reaction was monitored by ${ }^{1} \mathrm{H}-\mathrm{NMR}$. After completion of the acetate cleavage reaction, the reaction mixture was diluted with $50 \mathrm{~mL} \mathrm{H}_{2} \mathrm{O}$ and $100 \mathrm{~mL} \mathrm{CH}_{2} \mathrm{Cl}_{2}$. The organic layer was separated, and the aqueous layer was extracted with $\mathrm{CH}_{2} \mathrm{Cl}_{2}(3 \times 100$ $\mathrm{mL}$ ). The combined organic layer was dried over anhydrous $\mathrm{MgSO}_{4}$, and the solvent was removed under reduced pressure. Column chromatography on silica gel (elution with 1:3 ethyl acetate:hexanes) provided $570 \mathrm{mg}$ (79\% yield) of the diazo compound as a yellow oil that crystallized upon cooling: $\mathrm{mp} 39^{\circ} \mathrm{C}$; IR $(\mathrm{KBr}): 2117\left(\mathrm{C}=\mathrm{N}_{2}\right)$ and $1684(\mathrm{C}=\mathrm{O}) \mathrm{cm}^{-}$ ${ }^{1} ;{ }^{1} \mathrm{H}$ NMR $\left(250 \mathrm{MHz}, \mathrm{CDCl}_{3}\right.$ ): $\delta 6.28$ (ddd, $J=14.6,8.9,2.9 \mathrm{~Hz}, 2 \mathrm{H}$ ), 5.90-5.73 (comp, 2H), 4.78 (br s, 2H), 4.69 (d, $J=5.9 \mathrm{~Hz}, 4 \mathrm{H}) ;{ }^{13} \mathrm{C} \mathrm{NMR}\left(62.5 \mathrm{MHz}, \mathrm{CDCl}_{3}\right): \delta(\mathrm{C}=\mathrm{O}$ not observed), 132.8, 128.1, 64.6, 46.2; HRMS-FAB $(\mathrm{m} / \mathrm{z})$ : $\mathrm{M}^{+}$calcd for $\mathrm{C}_{10} \mathrm{H}_{10} \mathrm{~N}_{4} \mathrm{O}_{4}$, 250.0702; found, 250.0701 .

General Procedure for Dirhodium(II) Catalyzed Decomposition of E,E-2,4hexadien-1,6-diyl Bis-diazoacetate (5). The dirhodium(II) catalyst (1.0 mol \%, 0.0040

2. Bates, E. B.; Jones, E. R. H.; Witing, M. C. J. Chem. Soc. 1954, 147, 1794. 
mmol) was placed in an oven-dried, one-neck round bottom flask, equipped with a reflux condenser and a rubber septum and set under nitrogen. Then $5 \mathrm{~mL}$ of freshly distilled $\mathrm{CH}_{2} \mathrm{Cl}_{2}$ was added to dissolve the catalyst, and the resultant solution was stirred at reflux for $10 \mathrm{~min}$. The E,E-2,4-hexadien-1,6-diyl bis-diazoacetate $(100 \mathrm{mg}, 0.40 \mathrm{mmol})$ was dissolved in $10 \mathrm{~mL}$ of freshly distilled $\mathrm{CH}_{2} \mathrm{Cl}_{2}$ and added over $10 \mathrm{~h}$ via syringe pump (1.0 $\mathrm{mL} / \mathrm{h})$. Upon completion of the addition, the solution was heated at reflux for an additional $2 \mathrm{~h}$; then solvent was removed under reduced pressure. The product ratio was monitored by ${ }^{1} \mathrm{H}-\mathrm{NMR}$ in $\mathrm{CD}_{3} \mathrm{CN}$ of the crude reaction mixture. Column chromatography on silica gel (elution with $\mathrm{CHCl}_{3}$ ) provided a mixture of $\mathbf{6 a}$ and $\mathbf{6 b}$. The enantiomeric excess of $\mathbf{6 b}$ was determined by GC on a 30-m Chiraldex B-DM column: $t_{R}$ $77.7 \min \quad\left(\left(1 R, 5 S, 6 S, 1^{\prime} R, 5^{\prime} S, 6^{\prime} S\right)-6 \mathbf{b}\right), \quad 81.2 \quad \min \quad(\mathbf{6 a})$ and $91.2 \mathrm{~min}$ $\left(\left(1 S, 5 R, 6 R, 1^{\prime} S, 5^{\prime} R, 6^{\prime} R\right)-6 \mathbf{b}\right)$.

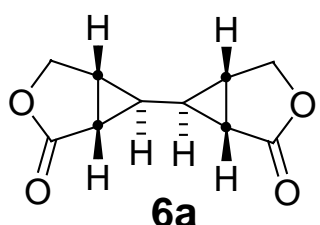

$\left(1 S, 5 R, 6 R, 1^{\prime} R, 5^{\prime} S, 6^{\prime} S\right)-\left[6,6^{\prime}\right] \mathrm{Bi}(3-0 x a-b i c y c l o[3.1 .0] h e x y l)-2,2^{\prime}$-dione $\quad$ (6a). An analytical sample of $\mathbf{6 a}$ was obtained by crystallization from $\mathrm{CHCl}_{3}$ of the reaction mixture that was obtained from the decomposition of 5 with $\mathrm{Rh}_{2}(+/-\mathrm{MEPY})_{4}$ : $\mathrm{mp}$ 224$226^{\circ} \mathrm{C}$ (decomposition); IR (KBr): $1743(\mathrm{C}=\mathrm{O}) \mathrm{cm}^{-1} ;{ }^{1} \mathrm{H}$ NMR $\left(250 \mathrm{MHz}, \mathrm{CDCl}_{3}\right): \delta 4.37$ $(\mathrm{dd}, J=9.5,4.8 \mathrm{~Hz}, 2 \mathrm{H}), 4.24(\mathrm{ddd}, J=9.5,0.8,0.8 \mathrm{~Hz}, 2 \mathrm{H}), 2.24-2.21$ (comp, 2H), 2.09 (dddd, $J=6.3,1.5,0.8,0.8 \mathrm{~Hz}, 2 \mathrm{H}), 1.15-1.08$ (comp, $2 \mathrm{H}$ ); ${ }^{13} \mathrm{C} \mathrm{NMR}\left(62.5 \mathrm{MHz}, \mathrm{CDCl}_{3}\right.$ ): $\delta 174.4,69.1,25.1,23.4,23.2$.

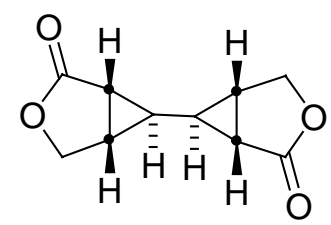

$(S)-6 \mathbf{b}$ 
$\left(1 R, 5 S, 6 S, 1^{\prime} R, 5^{\prime} S, 6^{\prime} S\right)$-[6,6']Bi(3-oxabicyclo[3.1.0]hexyl)-2,2'-dione $\quad(S-6 b) . \quad$ An analytical sample was obtained from the decomposition of 5 with $\mathrm{Rh}_{2}(4 S, S \text {-BSPIM })_{4}$, which contains $4 \%$ of $\mathbf{6 a}$ and $96 \%$ of $\mathbf{6 b}$ with $>99 \%$ ee: $\mathrm{mp} 139-140^{\circ} \mathrm{C} ;[\alpha]^{23}{ }_{\mathrm{D}}=+110.3$ $\left(c=0.41, \mathrm{CH}_{2} \mathrm{Cl}_{2}\right)$; IR (KBr): $1777(\mathrm{C}=\mathrm{O}) \mathrm{cm}^{-1} ;{ }^{1} \mathrm{H} \mathrm{NMR}\left(250 \mathrm{MHz}, \mathrm{CDCl}_{3}\right): \delta 4.36(\mathrm{dd}$, $J=9.5,4.8 \mathrm{~Hz}, 2 \mathrm{H}), 4.24$ (dd, $J=9.5,0.9 \mathrm{~Hz}, 2 \mathrm{H}), 2.21-2.16$ (comp, $2 \mathrm{H}$ ), 2.03 (dddd, $J$ $=6.3,1.5,0.8,0.8 \mathrm{~Hz}, 2 \mathrm{H}), 1.34-1.29$ (comp, $2 \mathrm{H}) ;{ }^{13} \mathrm{C} \mathrm{NMR}\left(125 \mathrm{MHz}, \mathrm{CDCl}_{3}\right): \delta 174.3$, 69.0, 24.7, 23.0, 22.8; LRMS-FAB $(\mathrm{m} / \mathrm{z}): 195[\mathrm{M}+\mathrm{H}]^{+}$.

\section{Crystal Structure Information for 2}

A colorless prism of $\mathrm{C} 10 \mathrm{H} 12 \mathrm{O} 4$, approximate dimensions $0.15 \times 0.27 \mathrm{X} 0.44 \mathrm{~mm}^{3}$, was used for the X-ray crystallographic analysis. The X-ray intensity data were measured at 213(2) $\mathrm{K}$ on a three-circle diffractometer system equipped with Bruker Smart1000 $\mathrm{CCD}$ area detector using a graphite monochromator and a MoK $\alpha$ fine-focus sealed tube $(\lambda=0.71073 \AA$ ) operated at $50 \mathrm{kV}$ and $40 \mathrm{~mA}$. The detector was placed at a distance of $4.99 \mathrm{~cm}$ from the crystal.

A total of 2791 frames were collected with a scan width of $0.3^{\circ}$ in $\omega$ and an exposure time of $15 \mathrm{sec} /$ frame using SMART (Bruker, 1999). The total data collection time was 17.2 hours. The frames were integrated with SAINT software package using a narrowframe integration algorithm. The integration of the data using a Monoclinic unit cell yielded a total of 10653 reflections to a maximum $\theta$ angle of $27.50^{\circ}$, of which 2143 were independent (completeness $=99.9 \%, \mathrm{R}_{\mathrm{int}}=3.07 \%, \mathrm{R}_{\mathrm{sig}}=1.70 \%$ ) and 2046 were greater than $2 \square(\mathrm{I})$. The final cell dimensions of $a=7.0222(4) \AA, b=12.2044(6) \AA, c=$ 10.4549(6) $\AA, \alpha=90^{\circ}, \beta=95.6310(10)^{\circ}, \gamma=90^{\circ}, V=891.68(8) \AA^{3}$, are based upon the refinement of the XYZ-centroids of 6754 reflections with $2.6<\theta<28.9^{\circ}$ using SAINT. Analysis of the data showed $0.27 \%$ decay during data collection. Data were corrected for absorption effects with the Semi-empirical from equivalents method using SADABS (Sheldrick, 1996). The minimum and maximum transmission coefficients were 0.905 and 0.983 . 
The structure was solved and refined using the SHELXS-97 (Sheldrick, 1990) and SHELXL-97 (Sheldrick, 1997) software in the space group $P 2_{1}$ with $Z=4$ for the formula unit $\mathrm{C} 10 \mathrm{H} 12 \mathrm{O} 4$. The final anisotropic full-matrix least-squares refinement on $\mathrm{F}^{2}$ with 350 variables converged at $\mathrm{R}_{1}=2.77 \%$ for the observed data and $\mathrm{wR}_{2}=5.94 \%$ for all data. The goodness-of-fit was 1.000. The largest peak on the final difference map was $0.194 \hat{\mathrm{e}} / \AA^{3}$ and the largest hole was $-0.133 \hat{\mathrm{e}} / \AA^{3}$. On the basis of the final model, the calculated density was $1.461 \mathrm{~g} / \mathrm{cm}^{3}$ and $\mathrm{F}(000)$, 416ê.

Table 1. Sample and crystal data for 2 .

\begin{tabular}{lll}
\hline X-ray labbook No. & 1247 \\
Empirical formula & $\mathrm{C} 10 \mathrm{H} 12 \mathrm{O} 4$ \\
Formula weight & 196.20 \\
Temperature & $213(2) \mathrm{K}$ \\
Wavelength & $0.71073 \AA$ \\
Crystal size & $0.44 \times 0.27 \times 0.15 \mathrm{~mm}^{3}$ \\
Crystal habit & colorless prism & \\
Crystal system & Monoclinic & \\
Space group & $P 2_{1}$ & \\
Unit cell dimensions & $a=7.0222(4) \AA$ & $\alpha=90^{\circ}$ \\
& $b=12.2044(6) \AA$ & $\beta=95.6310(10)^{\circ}$ \\
& $c=10.4549(6) \AA$ & $\gamma=90^{\circ}$ \\
Volume & $891.68(8) \AA^{3}$ & \\
$Z$ & 4 & \\
Density, $\rho_{\text {calc }}$ & $1.461 \mathrm{~g} / \mathrm{cm}^{3}$ & \\
Absorption coefficient, $\mu$ & $0.113 \mathrm{~mm}^{-1}$ & \\
F(000) & $416 \overline{\mathrm{e}}$ & \\
\hline
\end{tabular}

Table 2. Data collection and structure refinement for $\mathrm{C}_{10} \mathrm{H}_{12} \mathrm{O}_{4}$.

Diffractometer

Radiation source

Generator power

Detector distance

Detector resolution

Total frames

Frame size

Frame width

Exposure per frame
Bruker Smart1000 CCD area detector

fine-focus sealed tube, $\mathrm{MoK} \alpha$

$50 \mathrm{kV}, 40 \mathrm{ma}$

$4.99 \mathrm{~cm}$

8.33 pixels $/ \mathrm{mm}$

2791

512 pixels

$0.3^{\circ}$

$15 \mathrm{sec}$ 
Total measurement time

Data collection method

$\theta$ range for data collection

Index ranges

Reflections collected

Independent reflections

Observed reflection, $\mathrm{I}>2 \sigma(\mathrm{I})$

Coverage of independent reflections

Variation in check reflections

Absorption correction

Max. and min. transmission

Structure solution technique

Structure solution program

Refinement technique

Refinement program

Function minimized

Data / restraints / parameters

Goodness-of-fit on $\mathrm{F}^{2}$

$\Delta / \sigma_{\max }$

Final $R$ indices: $\quad R_{1}, \quad I>2 \sigma(I)$

$\mathrm{wR}_{2}$, all data

$\mathrm{R}_{\text {int }}$

$\mathrm{R}_{\text {sig }}$

Weighting scheme

Absolute structure parameter

Extinction coefficient

Largest diff. peak and hole
17.2 hours

$\omega$ and $\varphi$ scans

1.96 to $27.50^{\circ}$

$-9 \leq h \leq 9,-15 \leq k \leq 15,-13 \leq l \leq 13$

10653

2143

2046

$99.9 \%$

$0.27 \%$

Semi-empirical from equivalents

SADABS (Sheldrick, 1996)

0.983 and 0.905

direct

SHELXS-97 (Sheldrick, 1990)

Full-matrix least-squares on $\mathrm{F}^{2}$

SHELXL-97 (Sheldrick, 1997)

$\Sigma \mathrm{w}\left(\mathrm{F}_{\mathrm{o}}^{2}-\mathrm{F}_{\mathrm{c}}^{2}\right)^{2}$

2143 / 1 / 350

0.999

0.000

0.0277

0.0594

0.0307

0.0170

$\mathrm{W}=1 /\left[\sigma^{2}\left(\mathrm{~F}_{\mathrm{o}}^{2}\right)+(0.012 \mathrm{P})^{2}+0.2915 \mathrm{P}\right]$,

$\mathrm{P}=\left[\max \left(\mathrm{F}_{\mathrm{o}}^{2}, 0\right)+2 \mathrm{~F}_{\mathrm{o}}^{2}\right] / 3$

$-0.2(10)$

$0.059(3)$

0.194 and $-0.133 \overline{\mathrm{e}} / \AA^{3}$

$\mathrm{R}_{1}=\Sigma|| \mathrm{F}_{\mathrm{o}}|-| \mathrm{F}_{\mathrm{c}}|/ \Sigma| \mathrm{F}_{\mathrm{o}} \mid, \quad \mathrm{wR} 2=\left[\Sigma \mathrm{w}\left(\mathrm{F}_{\mathrm{o}}^{2}-\mathrm{F}_{\mathrm{c}}^{2}\right)^{2} / \Sigma \mathrm{w}\left(\mathrm{F}_{\mathrm{o}}^{2}\right)^{2}\right]^{1 / 2}$

Table 3. Atomic coordinates and equivalent ${ }^{*}$ isotropic atomic displacement parameters $\left(\AA^{2}\right)$ for 2 .

\begin{tabular}{ccccc}
\hline Atom & $x / a$ & $y / b$ & $z / c$ & $\mathrm{U}_{\text {eq }}$ \\
\hline O11 & $0.1306(2)$ & $0.17267(15)$ & $-0.09978(17)$ & $0.0487(4)$ \\
O12 & $0.4424(2)$ & $0.20709(12)$ & $-0.08722(15)$ & $0.0397(3)$ \\
C11 & $0.2923(3)$ & $0.13795(19)$ & $-0.09422(19)$ & $0.0352(5)$ \\
C12 & $0.3591(3)$ & $0.02159(17)$ & $-0.0950(2)$ & $0.0326(4)$ \\
C13 & $0.5770(3)$ & $0.02783(16)$ & $-0.09783(18)$ & $0.0286(4)$ \\
C14 & $0.6254(3)$ & $0.14910(17)$ & $-0.0658(2)$ & $0.0335(4)$ \\
C15 & $0.7076(3)$ & $0.16805(18)$ & $0.0722(2)$ & $0.0382(5)$ \\
C16 & $0.6199(3)$ & $0.08971(19)$ & $0.1636(2)$ & $0.0377(5)$
\end{tabular}




\begin{tabular}{lllll} 
C17 & $0.6704(3)$ & $-0.02865(18)$ & $0.13672(19)$ & $0.0333(4)$ \\
C18 & $0.6835(3)$ & $-0.05436(16)$ & $-0.00767(18)$ & $0.0300(4)$ \\
C19 & $0.8987(3)$ & $-0.06218(19)$ & $-0.0186(2)$ & $0.0348(4)$ \\
C20 & $0.9911(3)$ & $-0.06923(17)$ & $0.1168(2)$ & $0.0361(4)$ \\
O13 & $0.8612(2)$ & $-0.05115(14)$ & $0.20114(13)$ & $0.0386(3)$ \\
O14 & $1.1561(2)$ & $-0.08672(15)$ & $0.15355(16)$ & $0.0474(4)$ \\
O21 & $0.3421(2)$ & $0.85238(16)$ & $0.65520(17)$ & $0.0516(5)$ \\
O22 & $0.6509(2)$ & $0.81802(13)$ & $0.70389(14)$ & $0.0408(4)$ \\
C21 & $0.4979(3)$ & $0.83578(18)$ & $0.6194(2)$ & $0.0380(5)$ \\
C22 & $0.5514(3)$ & $0.8295(2)$ & $0.4844(2)$ & $0.0372(5)$ \\
C23 & $0.7696(3)$ & $0.82171(16)$ & $0.49629(19)$ & $0.0304(4)$ \\
C24 & $0.8237(3)$ & $0.79651(18)$ & $0.6403(2)$ & $0.0347(4)$ \\
C25 & $0.8821(3)$ & $0.6781(2)$ & $0.6678(2)$ & $0.0385(5)$ \\
C26 & $0.7662(3)$ & $0.59981(18)$ & $0.5784(2)$ & $0.0365(4)$ \\
C27 & $0.8056(3)$ & $0.61714(16)$ & $0.4398(2)$ & $0.0325(4)$ \\
C28 & $0.8459(3)$ & $0.73815(16)$ & $0.40566(18)$ & $0.0289(4)$ \\
C29 & $1.0635(3)$ & $0.74220(18)$ & $0.4031(2)$ & $0.0344(4)$ \\
C30 & $1.1275(3)$ & $0.62500(19)$ & $0.4031(2)$ & $0.0369(5)$ \\
O23 & $0.9796(2)$ & $0.55744(13)$ & $0.41735(16)$ & $0.0418(4)$ \\
O24 & $1.2858(2)$ & $0.58948(16)$ & $0.39408(17)$ & $0.0511(4)$ \\
\hline
\end{tabular}

${ }^{*} \mathrm{U}_{\mathrm{eq}}$ is defined as one third of the trace of the orthogonalized $\mathrm{U}_{\mathrm{ij}}$ tensor.

Table 4. Anisotropic atomic displacement parameters * $\left(\AA^{2}\right)$ for 2.

\begin{tabular}{|c|c|c|c|c|c|c|}
\hline Atom & $\mathrm{U}_{11}$ & $\mathrm{U}_{22}$ & $\mathrm{U}_{33}$ & $\mathrm{U}_{23}$ & $\mathrm{U}_{13}$ & $\mathrm{U}_{12}$ \\
\hline O11 & $0.0405(8)$ & $0.0491(10)$ & $0.0570(10)$ & $0.0097(8)$ & $0.0076(7)$ & $0.0126(8)$ \\
\hline $\mathrm{O} 12$ & $0.0431(8)$ & $0.0277(7)$ & $0.0479(9)$ & $0.0027(6)$ & $0.0029(6)$ & $0.0053(6)$ \\
\hline C11 & $0.0412(11)$ & $0.0365(11)$ & $0.0282(10)$ & $0.0050(8)$ & $0.0048(8)$ & $0.0043(9)$ \\
\hline $\mathrm{C} 12$ & $0.0346(10)$ & $0.0305(10)$ & $0.0325(10)$ & $0.0003(8)$ & $0.0031(8)$ & $-0.0013(8)$ \\
\hline C13 & $0.0335(10)$ & $0.0259(9)$ & $0.0269(9)$ & $-0.0008(7)$ & $0.0056(7)$ & $-0.0010(8)$ \\
\hline C14 & $0.0354(10)$ & $0.0260(10)$ & $0.0395(11)$ & $0.0027(8)$ & $0.0064(8)$ & $0.0001(8)$ \\
\hline $\mathrm{C} 15$ & $0.0415(11)$ & $0.0282(10)$ & $0.0442(11)$ & $-0.0079(9)$ & $0.0009(9)$ & $-0.0008(9)$ \\
\hline C16 & $0.0396(11)$ & $0.0434(12)$ & $0.0300(10)$ & $-0.0079(9)$ & $0.0035(8)$ & $0.0036(9)$ \\
\hline $\mathrm{C} 17$ & $0.0337(10)$ & $0.0371(11)$ & $0.0293(9)$ & $0.0044(8)$ & $0.0049(7)$ & $-0.0006(9)$ \\
\hline C18 & $0.0375(10)$ & $0.0241(9)$ & $0.0288(9)$ & $-0.0009(7)$ & $0.0060(7)$ & $-0.0018(8)$ \\
\hline C19 & $0.0376(10)$ & $0.0350(11)$ & $0.0326(10)$ & $0.0012(8)$ & $0.0076(8)$ & $0.0061(9)$ \\
\hline $\mathrm{C} 20$ & $0.0391(10)$ & $0.0319(11)$ & $0.0378(10)$ & $0.0060(9)$ & $0.0053(8)$ & $-0.0002(8)$ \\
\hline $\mathrm{O} 13$ & $0.0405(8)$ & $0.0449(9)$ & $0.0303(7)$ & $0.0052(6)$ & $0.0029(5)$ & $0.0038(7)$ \\
\hline O14 & $0.0362(8)$ & $0.0576(11)$ & $0.0477(9)$ & $0.0124(8)$ & $0.0014(6)$ & $0.0036(8)$ \\
\hline $\mathrm{O} 21$ & $0.0436(9)$ & $0.0607(11)$ & $0.0526(10)$ & $-0.0138(9)$ & $0.0145(7)$ & $0.0082(8)$ \\
\hline $\mathrm{O} 22$ & $0.0447(8)$ & $0.0456(9)$ & $0.0327(7)$ & $-0.0089(7)$ & $0.0075(6)$ & $0.0027(7)$ \\
\hline $\mathrm{C} 21$ & $0.0414(11)$ & $0.0311(11)$ & $0.0422(11)$ & $-0.0077(9)$ & $0.0074(9)$ & $0.0019(9)$ \\
\hline $\mathrm{C} 22$ & $0.0369(11)$ & $0.0392(12)$ & $0.0354(10)$ & $-0.0007(9)$ & $0.0031(8)$ & $0.0084(9)$ \\
\hline $\mathrm{C} 23$ & $0.0342(10)$ & $0.0241(9)$ & $0.0332(10)$ & $-0.0001(7)$ & $0.0046(8)$ & $-0.0004(8)$ \\
\hline $\mathrm{C} 24$ & $0.0346(10)$ & $0.0378(11)$ & $0.0316(10)$ & $-0.0073(9)$ & $0.0027(8)$ & $-0.0022(8)$ \\
\hline $\mathrm{C} 25$ & $0.0392(11)$ & $0.0456(12)$ & $0.0304(10)$ & $0.0051(9)$ & $0.0018(8)$ & $0.0045(9)$ \\
\hline $\mathrm{C} 26$ & $0.0390(11)$ & $0.0280(10)$ & $0.0433(11)$ & $0.0071(9)$ & $0.0085(9)$ & $0.0007(9)$ \\
\hline $\mathrm{C} 27$ & $0.0318(9)$ & $0.0274(10)$ & $0.0386(10)$ & $-0.0042(8)$ & $0.0041(8)$ & $-0.0005(8)$ \\
\hline
\end{tabular}




$\begin{array}{llllrrr}\text { C28 } & 0.0333(9) & 0.0273(9) & 0.0261(9) & 0.0001(7) & 0.0022(7) & 0.0003(7) \\ \text { C29 } & 0.0339(10) & 0.0319(10) & 0.0383(11) & 0.0021(9) & 0.0083(8) & -0.0008(8) \\ \text { C30 } & 0.0387(11) & 0.0378(12) & 0.0349(11) & -0.0019(9) & 0.0077(8) & 0.0044(9) \\ \text { O23 } & 0.0423(8) & 0.0285(8) & 0.0561(9) & -0.0027(7) & 0.0127(7) & 0.0051(6) \\ \text { O24 } & 0.0427(9) & 0.0515(11) & 0.0613(10) & -0.0009(8) & 0.0168(7) & 0.0130(8)\end{array}$

* The anisotropic atomic displacement factor exponent takes the form: $-2 \pi^{2}\left[h^{2} a^{* 2} U_{11}+\ldots+2 h k a * b * U_{12}\right]$

Table 5. Hydrogen atom coordinates and isotropic atomic displacement parameters $\left(\AA^{2}\right)$ for 2.

\begin{tabular}{lcccc}
\hline Atom & $x / a$ & $y / b$ & $z / c$ & $\mathrm{U}_{\text {iso }}$ \\
\hline H12A & $0.324(3)$ & $-0.015(2)$ & $-0.018(2)$ & $0.035(6)$ \\
H12B & $0.295(3)$ & $-0.019(2)$ & $-0.169(2)$ & $0.045(7)$ \\
H13 & $0.607(3)$ & $0.0121(19)$ & $-0.184(2)$ & $0.029(5)$ \\
H14 & $0.711(3)$ & $0.182(2)$ & $-0.127(2)$ & $0.037(6)$ \\
H15A & $0.850(3)$ & $0.155(2)$ & $0.077(2)$ & $0.036(6)$ \\
H15B & $0.685(3)$ & $0.245(2)$ & $0.093(2)$ & $0.037(6)$ \\
H16A & $0.478(3)$ & $0.096(2)$ & $0.156(2)$ & $0.036(6)$ \\
H16B & $0.661(3)$ & $0.1048(19)$ & $0.253(2)$ & $0.035(6)$ \\
H17 & $0.585(3)$ & $-0.079(2)$ & $0.173(2)$ & $0.035(6)$ \\
H18 & $0.627(3)$ & $-0.124(2)$ & $-0.027(2)$ & $0.030(5)$ \\
H19A & $0.950(4)$ & $0.001(2)$ & $-0.057(3)$ & $0.052(8)$ \\
H19B & $0.933(4)$ & $-0.126(3)$ & $-0.064(3)$ & $0.052(7)$ \\
H22A & $0.490(3)$ & $0.764(2)$ & $0.447(2)$ & $0.038(6)$ \\
H22B & $0.497(3)$ & $0.892(2)$ & $0.436(2)$ & $0.045(7)$ \\
H23 & $0.820(3)$ & $0.892(2)$ & $0.482(2)$ & $0.032(6)$ \\
H24 & $0.923(3)$ & $0.845(2)$ & $0.677(2)$ & $0.041(6)$ \\
H25A & $1.025(4)$ & $0.670(2)$ & $0.656(2)$ & $0.051(7)$ \\
H25B & $0.865(3)$ & $0.664(2)$ & $0.753(2)$ & $0.047(7)$ \\
H26A & $0.619(3)$ & $0.612(2)$ & $0.585(2)$ & $0.042(6)$ \\
H26B & $0.788(3)$ & $0.526(2)$ & $0.602(2)$ & $0.042(6)$ \\
H27 & $0.704(3)$ & $0.586(2)$ & $0.386(2)$ & $0.033(6)$ \\
H28 & $0.787(3)$ & $0.7521(19)$ & $0.321(2)$ & $0.027(5)$ \\
H29A & $1.128(4)$ & $0.777(2)$ & $0.481(3)$ & $0.047(7)$ \\
H29B & $1.110(4)$ & $0.782(2)$ & $0.331(3)$ & $0.050(7)$ \\
\hline
\end{tabular}

Table 6. Bond lengths $(\AA)$ and angles $\left(^{\circ}\right)$ for 2 .

\begin{tabular}{lllr}
\hline O11-C11 & $1.208(3)$ & O12-C11 & $1.347(3)$ \\
O12-C14 & $1.465(2)$ & $\mathrm{C} 11-\mathrm{C} 12$ & $1.496(3)$ \\
$\mathrm{C} 12-\mathrm{C} 13$ & $1.535(3)$ & $\mathrm{C} 12-\mathrm{H} 12 \mathrm{~A}$ & $0.97(2)$ \\
$\mathrm{C} 12-\mathrm{H} 12 \mathrm{~B}$ & $0.99(3)$ & $\mathrm{C} 13-\mathrm{C} 18$ & $1.521(3)$ \\
$\mathrm{C} 13-\mathrm{C} 14$ & $1.548(3)$ & $\mathrm{C} 13-\mathrm{H} 13$ & $0.97(2)$ \\
$\mathrm{C} 14-\mathrm{C} 15$ & $1.519(3)$ & $\mathrm{C} 14-\mathrm{H} 14$ & $1.00(2)$ \\
$\mathrm{C} 15-\mathrm{C} 16$ & $1.524(3)$ & $\mathrm{C} 15-\mathrm{H} 15 \mathrm{~A}$ & $1.01(2)$ \\
$\mathrm{C} 15-\mathrm{H} 15 \mathrm{~B}$ & $0.98(3)$ & $\mathrm{C} 16-\mathrm{C} 17$ & $1.520(3)$ \\
$\mathrm{C} 16-\mathrm{H} 16 \mathrm{~A}$ & $0.99(2)$ & $\mathrm{C} 16-\mathrm{H} 16 \mathrm{~B}$ & $0.97(2)$ \\
$\mathrm{C} 17-\mathrm{O} 13$ & $1.466(2)$ & $\mathrm{C} 17-\mathrm{C} 18$ & $1.553(3)$
\end{tabular}




\begin{tabular}{|c|c|c|c|}
\hline $\mathrm{C} 17-\mathrm{H} 17$ & $0.96(2)$ & C18-C19 & $1.530(3)$ \\
\hline C18-H18 & $0.96(2)$ & C19-C20 & $1.501(3)$ \\
\hline C19-H19A & $0.95(3)$ & C19-H19B & $0.96(3)$ \\
\hline $\mathrm{C} 20-\mathrm{O} 14$ & $1.203(3)$ & $\mathrm{C} 20-\mathrm{O} 13$ & $1.348(2)$ \\
\hline $\mathrm{O} 21-\mathrm{C} 21$ & $1.207(3)$ & $\mathrm{O} 22-\mathrm{C} 21$ & $1.340(3)$ \\
\hline $\mathrm{O} 22-\mathrm{C} 24$ & $1.464(2)$ & $\mathrm{C} 21-\mathrm{C} 22$ & $1.499(3)$ \\
\hline $\mathrm{C} 22-\mathrm{C} 23$ & $1.528(3)$ & $\mathrm{C} 22-\mathrm{H} 22 \mathrm{~A}$ & $0.98(3)$ \\
\hline $\mathrm{C} 22-\mathrm{H} 22 \mathrm{~B}$ & $0.97(3)$ & $\mathrm{C} 23-\mathrm{C} 28$ & $1.524(3)$ \\
\hline $\mathrm{C} 23-\mathrm{C} 24$ & $1.547(3)$ & $\mathrm{C} 23-\mathrm{H} 23$ & $0.95(2)$ \\
\hline $\mathrm{C} 24-\mathrm{C} 25$ & $1.521(3)$ & $\mathrm{C} 24-\mathrm{H} 24$ & $0.97(3)$ \\
\hline $\mathrm{C} 25-\mathrm{C} 26$ & $1.516(3)$ & $\mathrm{C} 25-\mathrm{H} 25 \mathrm{~A}$ & $1.02(3)$ \\
\hline $\mathrm{C} 25-\mathrm{H} 25 \mathrm{~B}$ & $0.93(3)$ & $\mathrm{C} 26-\mathrm{C} 27$ & $1.517(3)$ \\
\hline $\mathrm{C} 26-\mathrm{H} 26 \mathrm{~A}$ & $1.05(2)$ & $\mathrm{C} 26-\mathrm{H} 26 \mathrm{~B}$ & $0.94(3)$ \\
\hline $\mathrm{C} 27-\mathrm{O} 23$ & $1.461(2)$ & $\mathrm{C} 27-\mathrm{C} 28$ & $1.552(3)$ \\
\hline $\mathrm{C} 27-\mathrm{H} 27$ & $0.95(2)$ & C28-C29 & $1.532(3)$ \\
\hline $\mathrm{C} 28-\mathrm{H} 28$ & $0.96(2)$ & C29-C30 & $1.499(3)$ \\
\hline $\mathrm{C} 29-\mathrm{H} 29 \mathrm{~A}$ & $0.99(3)$ & $\mathrm{C} 29-\mathrm{H} 29 \mathrm{~B}$ & $0.98(3)$ \\
\hline $\mathrm{C} 30-\mathrm{O} 24$ & $1.205(3)$ & $\mathrm{C} 30-\mathrm{O} 23$ & $1.346(3)$ \\
\hline $\mathrm{C} 11-\mathrm{O} 12-\mathrm{C} 14$ & $112.07(17)$ & O11-C11-O12 & $120.7(2)$ \\
\hline O11-C11-C12 & $128.8(2)$ & O12-C11-C12 & $110.54(17)$ \\
\hline $\mathrm{C} 11-\mathrm{C} 12-\mathrm{C} 13$ & $105.48(17)$ & $\mathrm{C} 11-\mathrm{C} 12-\mathrm{H} 12 \mathrm{~A}$ & 109.1(14) \\
\hline $\mathrm{C} 13-\mathrm{C} 12-\mathrm{H} 12 \mathrm{~A}$ & $111.9(13)$ & C11-C12-H12B & $111.4(15)$ \\
\hline C13-C12-H12B & $112.7(14)$ & $\mathrm{H} 12 \mathrm{~A}-\mathrm{C} 12-\mathrm{H} 12 \mathrm{~B}$ & $106(2)$ \\
\hline C18-C13-C12 & $112.67(16)$ & C18-C13-C14 & $114.36(16)$ \\
\hline C12-C13-C14 & $103.95(16)$ & C18-C13-H13 & 108.1(13) \\
\hline C12-C13-H13 & $108.3(12)$ & C14-C13-H13 & $109.2(13)$ \\
\hline O12-C14-C15 & $108.51(17)$ & O12-C14-C13 & $104.98(15)$ \\
\hline C15-C14-C13 & $113.95(17)$ & O12-C14-H14 & $106.1(13)$ \\
\hline C15-C14-H14 & $110.3(13)$ & C13-C14-H14 & $112.5(14)$ \\
\hline C14-C15-C16 & $111.16(17)$ & C14-C15-H15A & $107.6(13)$ \\
\hline C16-C15-H15A & $109.3(14)$ & C14-C15-H15B & $107.5(14)$ \\
\hline $\mathrm{C} 16-\mathrm{C} 15-\mathrm{H} 15 \mathrm{~B}$ & $112.1(13)$ & H15A-C15-H15B & 109(2) \\
\hline C17-C16-C15 & $111.37(17)$ & C17-C16-H16A & $107.9(15)$ \\
\hline C15-C16-H16A & $111.2(14)$ & C17-C16-H16B & $107.8(14)$ \\
\hline $\mathrm{C} 15-\mathrm{C} 16-\mathrm{H} 16 \mathrm{~B}$ & $112.7(14)$ & H16A-C16-H16B & $105.6(18)$ \\
\hline O13-C17-C16 & $108.20(17)$ & O13-C17-C18 & $105.67(15)$ \\
\hline C16-C17-C18 & $114.13(17)$ & O13-C17-H17 & $106.4(14)$ \\
\hline $\mathrm{C} 16-\mathrm{C} 17-\mathrm{H} 17$ & $111.6(14)$ & $\mathrm{C} 18-\mathrm{C} 17-\mathrm{H} 17$ & $110.4(14)$ \\
\hline C13-C18-C19 & $115.07(16)$ & C13-C18-C17 & $113.37(16)$ \\
\hline C19-C18-C17 & $103.79(16)$ & C13-C18-H18 & $107.0(13)$ \\
\hline C19-C18-H18 & $108.7(13)$ & C17-C18-H18 & $108.8(14)$ \\
\hline C20-C19-C18 & $105.79(16)$ & C20-C19-H19A & $107.5(16)$ \\
\hline C18-C19-H19A & $113.6(16)$ & C20-C19-H19B & $108.3(16)$ \\
\hline C18-C19-H19B & $112.9(16)$ & H19A-C19-H19B & $108(2)$ \\
\hline $\mathrm{O} 14-\mathrm{C} 20-\mathrm{O} 13$ & $120.82(19)$ & $\mathrm{O} 14-\mathrm{C} 20-\mathrm{C} 19$ & $128.7(2)$ \\
\hline O13-C20-C19 & $110.48(18)$ & C20-O13-C17 & $112.16(15)$ \\
\hline $\mathrm{C} 21-\mathrm{O} 22-\mathrm{C} 24$ & $112.12(16)$ & $\mathrm{O} 21-\mathrm{C} 21-\mathrm{O} 22$ & $121.0(2)$ \\
\hline $\mathrm{O} 21-\mathrm{C} 21-\mathrm{C} 22$ & $128.3(2)$ & $\mathrm{O} 22-\mathrm{C} 21-\mathrm{C} 22$ & $110.71(17)$ \\
\hline $\mathrm{C} 21-\mathrm{C} 22-\mathrm{C} 23$ & $105.59(17)$ & $\mathrm{C} 21-\mathrm{C} 22-\mathrm{H} 22 \mathrm{~A}$ & $106.3(14)$ \\
\hline $\mathrm{C} 23-\mathrm{C} 22-\mathrm{H} 22 \mathrm{~A}$ & $112.4(14)$ & $\mathrm{C} 21-\mathrm{C} 22-\mathrm{H} 22 \mathrm{~B}$ & $109.3(14)$ \\
\hline
\end{tabular}




\begin{tabular}{lllr}
$\mathrm{C} 23-\mathrm{C} 22-\mathrm{H} 22 \mathrm{~B}$ & $115.4(15)$ & $\mathrm{H} 22 \mathrm{~A}-\mathrm{C} 22-\mathrm{H} 22 \mathrm{~B}$ & $107.5(19)$ \\
$\mathrm{C} 28-\mathrm{C} 23-\mathrm{C} 22$ & $113.68(16)$ & $\mathrm{C} 28-\mathrm{C} 23-\mathrm{C} 24$ & $113.84(16)$ \\
$\mathrm{C} 22-\mathrm{C} 23-\mathrm{C} 24$ & $103.92(16)$ & $\mathrm{C} 28-\mathrm{C} 23-\mathrm{H} 23$ & $110.2(14)$ \\
$\mathrm{C} 22-\mathrm{C} 23-\mathrm{H} 23$ & $108.5(14)$ & $\mathrm{C} 24-\mathrm{C} 23-\mathrm{H} 23$ & $106.2(14)$ \\
$\mathrm{O} 22-\mathrm{C} 24-\mathrm{C} 25$ & $107.72(18)$ & $\mathrm{O} 22-\mathrm{C} 24-\mathrm{C} 23$ & $105.73(16)$ \\
$\mathrm{C} 25-\mathrm{C} 24-\mathrm{C} 23$ & $114.11(17)$ & $\mathrm{O} 22-\mathrm{C} 24-\mathrm{H} 24$ & $107.7(15)$ \\
$\mathrm{C} 25-\mathrm{C} 24-\mathrm{H} 24$ & $109.8(15)$ & $\mathrm{C} 23-\mathrm{C} 24-\mathrm{H} 24$ & $111.4(15)$ \\
$\mathrm{C} 26-\mathrm{C} 25-\mathrm{C} 24$ & $111.34(17)$ & $\mathrm{C} 26-\mathrm{C} 25-\mathrm{H} 25 \mathrm{~A}$ & $109.9(15)$ \\
$\mathrm{C} 24-\mathrm{C} 25-\mathrm{H} 25 \mathrm{~A}$ & $108.2(17)$ & $\mathrm{H} 25 \mathrm{~A}-\mathrm{C} 25-\mathrm{H} 25 \mathrm{~B}$ & $111.4(16)$ \\
$\mathrm{C} 24-\mathrm{C} 25-\mathrm{H} 25 \mathrm{~B}$ & $107.5(17)$ & $\mathrm{C} 25-\mathrm{C} 26-\mathrm{H} 26 \mathrm{~A}$ & $108(2)$ \\
$\mathrm{C} 25-\mathrm{C} 26-\mathrm{C} 27$ & $111.30(17)$ & $\mathrm{C} 25-\mathrm{C} 26-\mathrm{H} 26 \mathrm{~B}$ & $109.9(14)$ \\
$\mathrm{C} 27-\mathrm{C} 26-\mathrm{H} 26 \mathrm{~A}$ & $108.4(13)$ & $\mathrm{H} 26 \mathrm{~A}-\mathrm{C} 26-\mathrm{H} 26 \mathrm{~B}$ & $112.2(15)$ \\
$\mathrm{C} 27-\mathrm{C} 26-\mathrm{H} 26 \mathrm{~B}$ & $109.9(15)$ & $\mathrm{O} 23-\mathrm{C} 27-\mathrm{C} 28$ & $105(2)$ \\
$\mathrm{O} 23-\mathrm{C} 27-\mathrm{C} 26$ & $108.54(16)$ & $\mathrm{O} 23-\mathrm{C} 27-\mathrm{H} 27$ & $105.27(15)$ \\
$\mathrm{C} 26-\mathrm{C} 27-\mathrm{C} 28$ & $114.03(16)$ & $\mathrm{C} 28-\mathrm{C} 27-\mathrm{H} 27$ & $107.3(13)$ \\
$\mathrm{C} 26-\mathrm{C} 27-\mathrm{H} 27$ & $108.4(13)$ & $\mathrm{C} 23-\mathrm{C} 28-\mathrm{C} 27$ & $113.0(14)$ \\
$\mathrm{C} 23-\mathrm{C} 28-\mathrm{C} 29$ & $113.63(16)$ & $\mathrm{C} 27-\mathrm{C} 28-\mathrm{H} 28$ & $114.24(15)$ \\
$\mathrm{C} 29-\mathrm{C} 28-\mathrm{C} 27$ & $103.85(16)$ & $\mathrm{C} 30-\mathrm{C} 29-\mathrm{H} 29 \mathrm{~A}$ & $108.1(13)$ \\
$\mathrm{C} 29-\mathrm{C} 28-\mathrm{H} 28$ & $108.7(12)$ & $\mathrm{C} 30-\mathrm{C} 29-\mathrm{H} 29 \mathrm{~B}$ & $108.1(14)$ \\
$\mathrm{C} 30-\mathrm{C} 29-\mathrm{C} 28$ & $105.59(17)$ & $\mathrm{H} 29 \mathrm{~A}-\mathrm{C} 29-\mathrm{H} 29 \mathrm{~B}$ & $107.5(16)$ \\
$\mathrm{C} 28-\mathrm{C} 29-\mathrm{H} 29 \mathrm{~A}$ & $111.9(15)$ & $\mathrm{O} 24-\mathrm{C} 30-\mathrm{C} 29$ & $110.7(16)$ \\
C28-C29-H29B & $115.7(15)$ & $\mathrm{C} 30-\mathrm{O} 23-\mathrm{C} 27$ & $128(2)$ \\
O24-C30-O23 & $121.1(2)$ & & $112.27(17)$ \\
O23-C30-C29 & $110.48(17)$ & & \\
\hline
\end{tabular}

Table 7. Torsion angles $\left({ }^{\circ}\right)$ for 2.

\begin{tabular}{lrrr}
\hline C14-O12-C11-O11 & $173.18(19)$ & C14-O12-C11-C12 & $-7.4(2)$ \\
O11-C11-C12-C13 & $175.2(2)$ & O12-C11-C12-C13 & $-4.2(2)$ \\
C11-C12-C13-C18 & $137.42(17)$ & C11-C12-C13-C14 & $13.1(2)$ \\
C11-O12-C14-C15 & $-106.55(19)$ & C11-O12-C14-C13 & $15.6(2)$ \\
C18-C13-C14-O12 & $-140.26(16)$ & C12-C13-C14-O12 & $-17.00(19)$ \\
C18-C13-C14-C15 & $-21.7(2)$ & C12-C13-C14-C15 & $101.59(19)$ \\
O12-C14-C15-C16 & $82.3(2)$ & C13-C14-C15-C16 & $-34.2(2)$ \\
C14-C15-C16-C17 & $64.2(2)$ & C15-C16-C17-O13 & $82.5(2)$ \\
C15-C16-C17-C18 & $-34.8(2)$ & C12-C13-C18-C19 & $172.61(17)$ \\
C14-C13-C18-C19 & $-69.0(2)$ & C12-C13-C18-C17 & $-68.1(2)$ \\
C14-C13-C18-C17 & $50.3(2)$ & O13-C17-C18-C13 & $-139.54(17)$ \\
C16-C17-C18-C13 & $-20.8(2)$ & O13-C17-C18-C19 & $-14.0(2)$ \\
C16-C17-C18-C19 & $104.8(2)$ & C13-C18-C19-C20 & $137.98(18)$ \\
C17-C18-C19-C20 & $13.5(2)$ & O14-C20-O13-C17 & $172.3(2)$ \\
C18-C19-C20-O13 & $-8.5(2)$ & C16-C17-O13-C20 & $-113.01(18)$ \\
C19-C20-O13-C17 & $-0.8(2)$ & C24-O22-C21-O21 & $178.3(2)$ \\
C18-C17-O13-C20 & $9.6(2)$ & O21-C21-C22-C23 & $172.9(2)$ \\
C24-O22-C21-C22 & $-0.7(2)$ & C21-C22-C23-C28 & $137.25(18)$ \\
O22-C21-C22-C23 & $-8.2(2)$ & C21-O22-C24-C25 & $-113.18(19)$ \\
C21-C22-C23-C24 & $13.0(2)$ & C28-C23-C24-O22 & $-137.60(17)$ \\
C21-O22-C24-C23 & $9.2(2)$ & C28-C23-C24-C25 & $-19.4(2)$ \\
C22-C23-C24-O22 & $-13.4(2)$ & O22-C24-C25-C26 & $81.1(2)$ \\
C22-C23-C24-C25 & $104.8(2)$ & &
\end{tabular}




\begin{tabular}{lccr} 
C23-C24-C25-C26 & $-35.9(2)$ & $\mathrm{C} 24-\mathrm{C} 25-\mathrm{C} 26-\mathrm{C} 27$ & $64.5(2)$ \\
$\mathrm{C} 25-\mathrm{C} 26-\mathrm{C} 27-\mathrm{O} 23$ & $82.9(2)$ & $\mathrm{C} 25-\mathrm{C} 26-\mathrm{C} 27-\mathrm{C} 28$ & $-34.1(2)$ \\
$\mathrm{C} 22-\mathrm{C} 23-\mathrm{C} 28-\mathrm{C} 29$ & $171.44(17)$ & $\mathrm{C} 24-\mathrm{C} 23-\mathrm{C} 28-\mathrm{C} 29$ & $-69.8(2)$ \\
$\mathrm{C} 22-\mathrm{C} 23-\mathrm{C} 28-\mathrm{C} 27$ & $-69.6(2)$ & $\mathrm{C} 24-\mathrm{C} 23-\mathrm{C} 28-\mathrm{C} 27$ & $49.1(2)$ \\
O23-C27-C28-C23 & $-140.17(16)$ & $\mathrm{C} 26-\mathrm{C} 27-\mathrm{C} 28-\mathrm{C} 23$ & $-21.3(2)$ \\
O23-C27-C28-C29 & $-15.9(2)$ & $\mathrm{C} 26-\mathrm{C} 27-\mathrm{C} 28-\mathrm{C} 29$ & $103.00(18)$ \\
$\mathrm{C} 23-\mathrm{C} 28-\mathrm{C} 29-\mathrm{C} 30$ & $137.86(18)$ & $\mathrm{C} 27-\mathrm{C} 28-\mathrm{C} 29-\mathrm{C} 30$ & $13.1(2)$ \\
$\mathrm{C} 28-\mathrm{C} 29-\mathrm{C} 30-\mathrm{O} 24$ & $175.3(2)$ & $\mathrm{C} 28-\mathrm{C} 29-\mathrm{C} 30-\mathrm{O} 23$ & $-5.8(2)$ \\
O24-C30-O23-C27 & $174.02(19)$ & $\mathrm{C} 29-\mathrm{C} 30-\mathrm{O} 23-\mathrm{C} 27$ & $-5.0(2)$ \\
$\mathrm{C} 26-\mathrm{C} 27-\mathrm{O} 23-\mathrm{C} 30$ & $-109.03(19)$ & $\mathrm{C} 28-\mathrm{C} 27-\mathrm{O} 23-\mathrm{C} 30$ & $13.4(2)$ \\
\hline
\end{tabular}

\section{Crystal Structure Information for 3}

A colorless prism of $\mathrm{C}_{10} \mathrm{H}_{12} \mathrm{O}_{4}$, approximate dimensions $0.18 \times 0.22 \times 0.26 \mathrm{~mm}^{3}$, was used for the $\mathrm{X}$-ray crystallographic analysis. The $\mathrm{X}$-ray intensity data were measured at 213(2) K on a three-circle diffractometer system equipped with Bruker Smart1000 $\mathrm{CCD}$ area detector using a graphite monochromator and a MoK $\alpha$ fine-focus sealed tube $(\lambda=0.71073 \AA$ ) operated at $50 \mathrm{kV}$ and $40 \mathrm{~mA}$. The detector was placed at a distance of $4.990 \mathrm{~cm}$ from the crystal.

A total of 1818 frames were collected with a scan width of $0.3000^{\circ}$ in $\omega$ and an exposure time of $15 \mathrm{sec} /$ frame using SMART (Bruker, 1999). The total data collection time was $\mathbf{1 0 . 5}$ hours. The frames were integrated with SAINT software package using a narrow-frame integration algorithm. The integration of the data using a Monoclinic unit cell yielded a total of 3682 reflections to a maximum $\theta$ angle of $27.49^{\circ}$, of which 1120 were independent (completeness $\left.=99.6 \%, \mathrm{R}_{\text {int }}=2.04 \%, \mathrm{R}_{\text {sig }}=1.65 \%\right)$ and 1012 were greater than $2 \sigma(\mathrm{I})$. The final cell dimensions of $a=5.3861(3) \AA, b=10.9679(7) \AA, c=$ 8.4107(5) $\AA, \alpha=90^{\circ}, \beta=107.8670(10)^{\circ}, \gamma=90^{\circ}, V=472.89(5) \AA^{3}$, are based upon the refinement of the XYZ-centroids of 1890 reflections with $2.5<\theta<28.3^{\circ}$ using SAINT. Analysis of the data showed $0.00 \%$ decay during data collection. Data were corrected for absorption effects with the Semi-empirical from equivalents method using SADABS (Sheldrick, 1996). The minimum and maximum transmission coefficients were 0.901 and 0.981 .

The structure was solved and refined using the SHELXS-97 (Sheldrick, 1990) and SHELXL-97 (Sheldrick, 1997) software in the space group $P 2_{1}$ with $Z=2$ for the formula unit $\mathrm{C}_{10} \mathrm{H}_{12} \mathrm{O}_{4}$. The final anisotropic full-matrix least-squares refinement on $\mathrm{F}^{2}$ 
with 175 variables converged at $\mathrm{R}_{1}=3.54 \%$ for the observed data and $\mathrm{wR}_{2}=7.82 \%$ for all data. The goodness-of-fit was 1.000. The largest peak on the final difference map was $0.224 \overline{\mathrm{e}} / \AA^{3}$ and the largest hole was $-0.148 \overline{\mathrm{e}} / \AA^{3}$. On the basis of the final model, the calculated density was $1.378 \mathrm{~g} / \mathrm{cm}^{3}$ and $\mathrm{F}(000), 208 \overline{\mathrm{e}}$.

Table 1. Sample and crystal data for 3.

\begin{tabular}{lll}
\hline X-ray labbook No. & 1240 \\
Empirical formula & $\mathrm{C}_{10} \mathrm{H}_{12} \mathrm{O}_{4}$ & \\
Formula weight & 196.20 \\
Temperature & $213(2) \mathrm{K}$ \\
Wavelength & $0.71073 \AA$ \\
Crystal size & $0.26 \times 0.22 \times 0.18 \mathrm{~mm}^{3}$ \\
Crystal habit & colorless prism & \\
Crystal system & Monoclinic & \\
Space group & $P 2_{1}$ & \\
Unit cell dimensions & $a=5.3861(3) \AA$ & $\alpha=90^{\circ}$ \\
& $b=10.9679(7) \AA$ & $\beta=107.8670(10)^{\circ}$ \\
& $c=8.4107(5) \AA$ & $\gamma=90^{\circ}$ \\
Volume & $472.89(5) \AA^{3}$ & \\
$Z$ & 2 & \\
Density, $\rho_{\text {calc }}$ & $1.378 \mathrm{~g} / \mathrm{cm}^{3}$ & \\
Absorption coefficient, $\mu$ & $0.107 \mathrm{~mm}^{-1}$ & \\
F(000) & $208 \overline{\mathrm{e}}$ & \\
\hline
\end{tabular}

Table 2. Data collection and structure refinement for 3.

$\begin{array}{ll}\text { Diffractometer } & \text { Bruker Smart1000 CCD area detector } \\ \text { Radiation source } & \text { fine-focus sealed tube, MoK } \alpha \\ \text { Generator power } & 50 \mathrm{kV}, 40 \mathrm{ma} \\ \text { Detector distance } & 4.99 \mathrm{~cm} \\ \text { Detector resolution } & 8.33 \text { pixels } / \mathrm{mm} \\ \text { Total frames } & 1818 \\ \text { Frame size } & 512 \text { pixels } \\ \text { Frame width } & 0.3^{\circ} \\ \text { Exposure per frame } & 15 \mathrm{sec} \\ \text { Total measurement time } & 10.5 \text { hours } \\ \text { Data collection method } & \omega \text { scans } \\ \theta \text { range for data collection } & 3.15 \text { to } 27.49^{\circ} \\ \text { Index ranges } & -6 \leq h \leq 6,-14 \leq k \leq 14,-10 \leq l \leq 10\end{array}$


Reflections collected

3682

Independent reflections

Observed reflection, $\mathrm{I}>2 \sigma(\mathrm{I})$

Coverage of independent reflections

Variation in check reflections

Absorption correction

Max. and min. transmission

Structure solution technique

Structure solution program

Refinement technique

Refinement program

Function minimized

Data / restraints / parameters

Goodness-of-fit on $\mathrm{F}^{2}$

$\Delta / \sigma_{\max }$

Final $\mathrm{R}$ indices: $\quad \mathrm{R}_{1}, \quad \mathrm{I}>2 \sigma(\mathrm{I})$

$\mathrm{wR}_{2}$, all data

$\mathrm{R}_{\text {int }}$

$\mathrm{R}_{\text {sig }}$

Weighting scheme

Absolute structure parameter

Largest diff. peak and hole
1120

1012

$99.6 \%$

$0.00 \%$

Semi-empirical from equivalents

SADABS (Sheldrick, 1996)

0.981 and 0.901

direct

SHELXS-97 (Sheldrick, 1990)

Full-matrix least-squares on $\mathrm{F}^{2}$

SHELXL-97 (Sheldrick, 1997)

$\mathrm{SW}\left(\mathrm{F}_{\mathrm{o}}^{2}-\mathrm{F}_{\mathrm{c}}^{2}\right)^{2}$

$1120 / 1 / 175$

1.000

0.000

0.0354

0.0782

0.0204

0.0165

$\mathrm{w}=1 /\left[\sigma^{2}\left(\mathrm{~F}_{\mathrm{o}}^{2}\right)+(0.022 \mathrm{P})^{2}+0.177 \mathrm{P}\right]$,

$\mathrm{P}=\left[\max \left(\mathrm{F}_{\mathrm{o}}^{2}, 0\right)+2 \mathrm{~F}_{\mathrm{o}}^{2}\right] / 3$

$0.5(18)$

0.224 and $-0.148 \overline{\mathrm{e}} / \AA^{3}$

$\mathrm{R}_{1}=\Sigma|| \mathrm{F}_{\mathrm{o}}|-| \mathrm{F}_{\mathrm{c}}|/ \Sigma| \mathrm{F}_{\mathrm{o}} \mid, \quad \mathrm{wR} 2=\left[\Sigma \mathrm{w}\left(\mathrm{F}_{\mathrm{o}}^{2}-\mathrm{F}_{\mathrm{c}}^{2}\right)^{2} / \Sigma \mathrm{w}\left(\mathrm{F}_{\mathrm{o}}^{2}\right)^{2}\right]^{1 / 2}$ 
Table 3. Atomic coordinates and equivalent ${ }^{*}$ isotropic atomic displacement parameters $\left(\AA^{2}\right)$ for $\mathbf{3}$.

\begin{tabular}{lclcc}
\hline Atom & $x / a$ & $y / b$ & $z / c$ & $\mathrm{U}_{\text {eq }}$ \\
\hline O1 & $0.4351(4)$ & $0.0000(2)$ & $0.0118(3)$ & $0.0685(7)$ \\
O2 & $0.4490(3)$ & $0.15270(19)$ & $0.1973(2)$ & $0.0513(5)$ \\
C1 & $0.3312(5)$ & $0.0706(3)$ & $0.0768(3)$ & $0.0485(6)$ \\
C2 & $0.0567(5)$ & $0.1045(3)$ & $0.0702(4)$ & $0.0480(6)$ \\
C3 & $0.1870(5)$ & $0.1907(2)$ & $0.2136(3)$ & $0.0424(6)$ \\
C4 & $0.1455(7)$ & $0.3249(3)$ & $0.1823(4)$ & $0.0561(7)$ \\
C5 & $0.3337(8)$ & $0.4013(3)$ & $0.3169(4)$ & $0.0618(8)$ \\
C6 & $0.3612(6)$ & $0.3606(2)$ & $0.4929(4)$ & $0.0508(7)$ \\
C7 & $0.3776(5)$ & $0.2241(2)$ & $0.5236(3)$ & $0.0446(6)$ \\
C8 & $0.1773(5)$ & $0.1554(2)$ & $0.3842(3)$ & $0.0409(5)$ \\
C9 & $0.3108(6)$ & $0.2168(3)$ & $0.6858(4)$ & $0.0517(7)$ \\
C10 & $0.1120(5)$ & $0.3172(3)$ & $0.6648(3)$ & $0.0498(6)$ \\
O3 & $0.1300(4)$ & $0.3946(2)$ & $0.5443(2)$ & $0.0558(5)$ \\
O4 & $-0.0462(4)$ & $0.3309(2)$ & $0.7386(2)$ & $0.0628(6)$ \\
\hline
\end{tabular}

${ }^{*} \mathrm{U}_{\mathrm{eq}}$ is defined as one third of the trace of the orthogonalized $\mathrm{U}_{\mathrm{ij}}$ tensor.

Table 4. Anisotropic atomic displacement parameters * $\left(\AA^{2}\right)$ for 3.

\begin{tabular}{lllccc}
\hline Atom & \multicolumn{1}{c}{$\mathrm{U}_{11}$} & $\mathrm{U}_{22}$ & $\mathrm{U}_{33}$ & $\mathrm{U}_{23}$ & $\mathrm{U}_{13}$ \\
\hline O1 & $0.0640(14)$ & $0.0699(15)$ & $0.0813(15)$ & $-0.0184(13)$ & $0.0366(11) 0.0016(12)$ \\
O2 & $0.0417(9)$ & $0.0529(11)$ & $0.0621(11)$ & $-0.0080(10)$ & $0.0200(8)-0.0045(9)$ \\
C1 & $0.0528(15)$ & $0.0484(15)$ & $0.0491(14)$ & $-0.0039(12)$ & $0.0228(12)-0.0042(13)$ \\
C2 & $0.0452(14)$ & $0.0488(15)$ & $0.0499(15)$ & $-0.0084(13)$ & $0.0146(12)-0.0024(13)$ \\
C3 & $0.0418(12)$ & $0.0369(13)$ & $0.0486(13)$ & $-0.0011(11)$ & $0.0139(10)-0.0003(10)$ \\
C4 & $0.076(2)$ & $0.0399(15)$ & $0.0502(15)$ & $0.0090(13)$ & $0.0158(14) 0.0061(14)$ \\
C5 & $0.088(2)$ & $0.0329(14)$ & $0.0670(19)$ & $0.0036(14)$ & $0.0280(17)-0.0106(16)$ \\
C6 & $0.0526(15)$ & $0.0406(14)$ & $0.0558(15)$ & $-0.0051(12)$ & $0.0115(13)-0.0064(12)$ \\
C7 & $0.0446(14)$ & $0.0398(14)$ & $0.0478(14)$ & $0.0016(12)$ & $0.0117(11) 0.0032(11)$ \\
C8 & $0.0473(13)$ & $0.0292(12)$ & $0.0502(13)$ & $0.0014(10)$ & $0.0208(11)-0.0008(10)$ \\
C9 & $0.0558(16)$ & $0.0527(17)$ & $0.0452(14)$ & $0.0060(14)$ & $0.0133(12) 0.0110(14)$ \\
C10 & $0.0476(14)$ & $0.0546(17)$ & $0.0412(13)$ & $-0.0094(13)$ & $0.0047(11) 0.0043(13)$ \\
O3 & $0.0642(12)$ & $0.0471(10)$ & $0.0541(11)$ & $-0.0036(9)$ & $0.0151(9) 0.0138(10)$ \\
O4 & $0.0530(11)$ & $0.0804(16)$ & $0.0549(11)$ & $-0.0166(11)$ & $0.0163(9) 0.0028(11)$ \\
\hline
\end{tabular}

* The anisotropic atomic displacement factor exponent takes the form: $-2 \pi^{2}\left[h^{2} a^{* 2} U_{11}+\ldots+2 h k a * b * U_{12}\right]$ 
Table 5. Hydrogen atom coordinates and isotropic atomic displacement parameters $\left(\AA^{2}\right)$ for 3 .

\begin{tabular}{lcccc}
\hline Atom & $x / a$ & $y / b$ & $z / c$ & $\mathrm{U}_{\text {iso }}$ \\
\hline H21 & $-0.033(6)$ & $0.148(3)$ & $-0.031(4)$ & $0.051(8)$ \\
H22 & $-0.038(5)$ & $0.039(3)$ & $0.096(3)$ & $0.048(8)$ \\
H41 & $0.173(6)$ & $0.347(3)$ & $0.063(4)$ & $0.066(10)$ \\
H42 & $-0.036(7)$ & $0.336(3)$ & $0.178(4)$ & $0.065(10)$ \\
H51 & $0.512(7)$ & $0.390(4)$ & $0.308(4)$ & $0.068(10)$ \\
H52 & $0.272(6)$ & $0.490(4)$ & $0.309(4)$ & $0.071(9)$ \\
H61 & $0.519(6)$ & $0.401(3)$ & $0.571(3)$ & $0.050(8)$ \\
H71 & $0.568(6)$ & $0.189(3)$ & $0.530(4)$ & $0.059(9)$ \\
H81 & $0.203(5)$ & $0.069(3)$ & $0.398(3)$ & $0.041(7)$ \\
H82 & $0.003(6)$ & $0.175(3)$ & $0.392(4)$ & $0.055(8)$ \\
H91 & $0.236(5)$ & $0.139(3)$ & $0.709(3)$ & $0.047(8)$ \\
H92 & $0.454(6)$ & $0.238(3)$ & $0.782(4)$ & $0.061(9)$ \\
\hline
\end{tabular}

Table 6. Bond lengths $(\AA)$, valence and torsion angles $\left(^{\circ}\right)$ for $\mathbf{3}$.

\begin{tabular}{lclcl}
\hline $\mathrm{O} 1-\mathrm{C} 1$ & $1.182(3)$ & $\mathrm{O} 2-\mathrm{C} 1$ & $1.359(3)$ & $\mathrm{O} 2-\mathrm{C} 3$ \\
$1.518(3)$ & $\mathrm{C} 1-\mathrm{C} 2$ & $1.509(4)$ & $\mathrm{C} 2-\mathrm{C} 3$ & $1.524(4)$ \\
$\mathrm{C} 2-\mathrm{H} 21$ & $0.96(3)$ & $\mathrm{C} 2-\mathrm{H} 22$ & $0.94(3)$ & $\mathrm{C} 3-\mathrm{C} 4$ \\
$1.499(4)$ & $\mathrm{C} 3-\mathrm{C} 8$ & $1.502(4)$ & $\mathrm{C} 4-\mathrm{C} 5$ & $1.519(5)$ \\
$\mathrm{C} 4-\mathrm{H} 41$ & $1.08(3)$ & $\mathrm{C} 4-\mathrm{H} 42$ & $0.97(3)$ & $\mathrm{C} 5-\mathrm{C} 6$ \\
$1.509(4)$ & $\mathrm{C} 5-\mathrm{H} 51$ & $0.99(3)$ & $\mathrm{C} 5-\mathrm{H} 52$ & $1.03(4)$ \\
$\mathrm{C} 6-\mathrm{O} 3$ & $1.486(4)$ & $\mathrm{C} 6-\mathrm{C} 7$ & $1.517(4)$ & $\mathrm{C} 6-\mathrm{H} 61$ \\
$1.01(3)$ & $\mathrm{C} 7-\mathrm{C} 9$ & $1.516(4)$ & $\mathrm{C} 7-\mathrm{C} 8$ & $1.527(4)$ \\
$\mathrm{C} 7-\mathrm{H} 71$ & $1.08(3)$ & $\mathrm{C} 8-\mathrm{H} 81$ & $0.97(3)$ & $\mathrm{C} 8-\mathrm{H} 82$ \\
$0.98(3)$ & $\mathrm{C} 9-\mathrm{C} 10$ & $1.508(4)$ & $\mathrm{C} 9-\mathrm{H} 91$ & $0.99(3)$ \\
$\mathrm{C} 9-\mathrm{H} 92$ & $0.96(3)$ & $\mathrm{C} 10-\mathrm{O} 4$ & $1.207(3)$ & $\mathrm{C} 10-\mathrm{O} 3$ \\
$1.348(4)$ & & & & \\
& & & & \\
$\mathrm{C} 1-\mathrm{O} 2-\mathrm{C} 3$ & $90.90(18)$ & $\mathrm{O} 1-\mathrm{C} 1-\mathrm{O} 2$ & $126.9(3)$ & $\mathrm{O} 1-\mathrm{C} 1-\mathrm{C} 2$ \\
$137.9(3)$ & $\mathrm{O} 2-\mathrm{C} 1-\mathrm{C} 2$ & $95.3(2)$ & $\mathrm{C} 1-\mathrm{C} 2-\mathrm{C} 3$ & $85.2(2)$ \\
$\mathrm{C} 1-\mathrm{C} 2-\mathrm{H} 21$ & $112.2(18)$ & $\mathrm{C} 3-\mathrm{C} 2-\mathrm{H} 21$ & $112(2)$ & $\mathrm{C} 1-\mathrm{C} 2-\mathrm{H} 22$ \\
$113.6(18)$ & $\mathrm{C} 3-\mathrm{C} 2-\mathrm{H} 22$ & $116.0(17)$ & $\mathrm{H} 21-\mathrm{C} 2-\mathrm{H} 22$ & $114(3)$ \\
$\mathrm{C} 4-\mathrm{C} 3-\mathrm{C} 8$ & $112.0(2)$ & $\mathrm{C} 4-\mathrm{C} 3-\mathrm{O} 2$ & $110.3(2)$ & $\mathrm{C} 8-\mathrm{C} 3-\mathrm{O} 2$ \\
$108.7(2)$ & $\mathrm{C} 4-\mathrm{C} 3-\mathrm{C} 2$ & $117.5(2)$ & $\mathrm{C} 8-\mathrm{C} 3-\mathrm{C} 2$ & $117.0(2)$ \\
$\mathrm{O} 2-\mathrm{C} 3-\mathrm{C} 2$ & $88.43(18)$ & $\mathrm{C} 3-\mathrm{C} 4-\mathrm{C} 5$ & $112.4(3)$ & $\mathrm{C} 3-\mathrm{C} 4-\mathrm{H} 41$ \\
$109.3(19)$ & $\mathrm{C} 5-\mathrm{C} 4-\mathrm{H} 41$ & $108.4(17)$ & $\mathrm{C} 3-\mathrm{C} 4-\mathrm{H} 42$ & $103(2)$ \\
$\mathrm{C} 5-\mathrm{C} 4-\mathrm{H} 42$ & $112.8(19)$ & $\mathrm{H} 41-\mathrm{C} 4-\mathrm{H} 42$ & $111(3)$ & $\mathrm{C} 6-\mathrm{C} 5-\mathrm{C} 4$ \\
$114.2(3)$ & $\mathrm{C} 6-\mathrm{C} 5-\mathrm{H} 51$ & $103(2)$ & $\mathrm{C} 4-\mathrm{C} 5-\mathrm{H} 51$ & $108(2)$ \\
$\mathrm{C} 6-\mathrm{C} 5-\mathrm{H} 52$ & $106.1(18)$ & $\mathrm{C} 4-\mathrm{C} 5-\mathrm{H} 52$ & $110.3(19)$ & $\mathrm{H} 51-\mathrm{C} 5-\mathrm{H} 52$ \\
$114(3)$ & $\mathrm{O} 3-\mathrm{C} 6-\mathrm{C} 5$ & $111.9(3)$ & $\mathrm{O} 3-\mathrm{C} 6-\mathrm{C} 7$ & $102.0(2)$ \\
$\mathrm{C} 5-\mathrm{C} 6-\mathrm{C} 7$ & $116.2(3)$ & $\mathrm{O} 3-\mathrm{C} 6-\mathrm{H} 61$ & $108.3(16)$ & $\mathrm{C} 5-\mathrm{C} 6-\mathrm{H} 61$ \\
$108.7(17)$ & $\mathrm{C} 7-\mathrm{C} 6-\mathrm{H} 61$ & $109.4(18)$ & $\mathrm{C} 9-\mathrm{C} 7-\mathrm{C} 6$ & $100.8(2)$ \\
$\mathrm{C} 9-\mathrm{C} 7-\mathrm{C} 8$ & $111.0(2)$ & $\mathrm{C} 6-\mathrm{C} 7-\mathrm{C} 8$ & $111.5(2)$ & $\mathrm{C} 9-\mathrm{C} 7-\mathrm{H} 71$ \\
$114.7(17)$ & $\mathrm{C6-C7-H71}$ & $111.5(17)$ & $\mathrm{C} 8-\mathrm{C} 7-\mathrm{H} 71$ & $107.4(17)$ \\
$\mathrm{C} 3-\mathrm{C} 8-\mathrm{C} 7$ & $112.5(2)$ & $\mathrm{C} 3-\mathrm{C} 8-\mathrm{H} 81$ & $109.1(16)$ & $\mathrm{C} 7-\mathrm{C} 8-\mathrm{H} 81$ \\
& & & &
\end{tabular}




\begin{tabular}{|c|c|c|c|c|c|}
\hline $110.5(16)$ & C3-C8-H82 & $108.6(17)$ & C7-C8-H82 & $107.9(17)$ & \\
\hline H81-C8-H82 & $108(3)$ & $\mathrm{C} 10-\mathrm{C} 9-\mathrm{C} 7$ & $102.2(2)$ & C10-C9-H91 & \\
\hline 109.6(16) & C7-C9-H91 & $116.4(16)$ & C10-C9-H92 & $106(2)$ & \\
\hline C7-C9-H92 & $113.3(19)$ & H91-C9-H92 & $109(3)$ & $\mathrm{O} 4-\mathrm{C} 10-\mathrm{O} 3$ & \\
\hline $122.2(3)$ & $\mathrm{O} 4-\mathrm{C} 10-\mathrm{C} 9$ & $128.2(3)$ & $\mathrm{O} 3-\mathrm{C} 10-\mathrm{C} 9$ & $109.6(2)$ & \\
\hline $\mathrm{C} 10-\mathrm{O} 3-\mathrm{C} 6$ & $108.7(2)$ & & & & \\
\hline $\mathrm{C} 3-\mathrm{O} 2-\mathrm{C} 1-\mathrm{O} 1$ & $-175.3(3)$ & $\mathrm{C} 3-\mathrm{O} 2-\mathrm{C} 1-\mathrm{C} 2$ & $3.7(2)$ & $\mathrm{O} 1-\mathrm{C} 1-\mathrm{C} 2-\mathrm{C} 3$ & \\
\hline $175.1(4)$ & $\mathrm{O} 2-\mathrm{C} 1-\mathrm{C} 2-\mathrm{C} 3$ & $-3.7(2)$ & $\mathrm{C} 1-\mathrm{O} 2-\mathrm{C} 3-\mathrm{C} 4$ & $-122.5(2)$ & \\
\hline $\mathrm{C} 1-\mathrm{O} 2-\mathrm{C} 3-\mathrm{C} 8$ & $114.4(2)$ & $\mathrm{C} 1-\mathrm{O} 2-\mathrm{C} 3-\mathrm{C} 2$ & $-3.6(2)$ & C1-C2-C3-C4 & \\
\hline $115.4(3)$ & $\mathrm{C} 1-\mathrm{C} 2-\mathrm{C} 3-\mathrm{C} 8$ & $-107.0(2)$ & $\mathrm{C} 1-\mathrm{C} 2-\mathrm{C} 3-\mathrm{O} 2$ & $3.3(2)$ & \\
\hline $\mathrm{C} 8-\mathrm{C} 3-\mathrm{C} 4-\mathrm{C} 5$ & $54.2(4)$ & $\mathrm{O} 2-\mathrm{C} 3-\mathrm{C} 4-\mathrm{C} 5$ & $-67.0(3)$ & $\mathrm{C} 2-\mathrm{C} 3-\mathrm{C} 4-\mathrm{C} 5$ & - \\
\hline $166.2(3)$ & C3-C4-C5-C6 & $-46.2(4)$ & $\mathrm{C} 4-\mathrm{C} 5-\mathrm{C} 6-\mathrm{O} 3$ & $-74.6(4)$ & \\
\hline C4-C5-C6-C7 & $42.0(4)$ & $\mathrm{O} 3-\mathrm{C} 6-\mathrm{C} 7-\mathrm{C} 9$ & $-39.7(3)$ & C5-C6-C7-C9 & - \\
\hline $161.6(3)$ & O3-C6-C7-C8 & $78.2(3)$ & C5-C6-C7-C8 & $-43.8(4)$ & \\
\hline C4-C3-C8-C7 & $-57.5(3)$ & $\mathrm{O} 2-\mathrm{C} 3-\mathrm{C} 8-\mathrm{C} 7$ & $64.6(3)$ & C2-C3-C8-C7 & \\
\hline $162.7(2)$ & C9-C7-C8-C3 & $162.6(2)$ & $\mathrm{C} 6-\mathrm{C} 7-\mathrm{C} 8-\mathrm{C} 3$ & $51.1(3)$ & \\
\hline C6-C7-C9-C10 & $35.4(3)$ & C8-C7-C9-C10 & $-82.8(3)$ & C7-C9-C10-O4 & \\
\hline $160.7(3)$ & C7-C9-C10-O3 & $-18.6(3)$ & $\mathrm{O} 4-\mathrm{C} 10-\mathrm{O} 3-\mathrm{C} 6$ & $173.6(3)$ & \\
\hline $\begin{array}{l}\text { C9-C10-O3-C6 } \\
30.0(3)\end{array}$ & $-7.1(3)$ & C5-C6-O3-C10 & $154.9(2)$ & C7-C6-O3-C10 & \\
\hline
\end{tabular}

\section{Crystal Structure Information for 4}

A colorless needle of $\mathrm{C}_{10} \mathrm{H}_{12} \mathrm{O}_{4}$, approximate dimensions $0.08 \times 0.18 \times 0.45 \mathrm{~mm}^{3}$, was used for the X-ray crystallographic analysis. The X-ray intensity data were measured at $213(2) \mathrm{K}$ on a three-circle diffractometer system equipped with Bruker Smart1000 CCD area detector using a graphite monochromator and a MoK $\alpha$ fine-focus sealed tube $(\lambda=0.71073 \AA$ ) operated at $50 \mathrm{kV}$ and $40 \mathrm{~mA}$. The detector was placed at a distance of $4.99 \mathrm{~cm}$ from the crystal.

A total of 3124 frames were collected with a scan width of $0.3^{\circ}$ in $\omega$ and an exposure time of $15 \mathrm{sec} /$ frame using SMART (Bruker, 1999). The total data collection time was 19.2 hours. The frames were integrated with SAINT software package using a narrowframe integration algorithm. The integration of the data using a Monoclinic unit cell yielded a total of 5725 reflections to a maximum $\theta$ angle of $27.49^{\circ}$, of which 1058 were independent (completeness $\left.=99.9 \%, \mathrm{R}_{\text {int }}=2.00 \%, \mathrm{R}_{\text {sig }}=1.31 \%\right)$ and 962 were greater than $2 \sigma(\mathrm{I})$. The final cell dimensions of $a=8.4475(8) \AA, b=5.4646(5) \AA, c=10.2032(9)$ $\AA, \alpha=90^{\circ}, \beta=99.118(2)^{\circ}, \gamma=90^{\circ}, V=465.05(7) \AA^{3}$, are based upon the refinement of the 
XYZ-centroids of 3031 reflections with $2.9<\theta<28.9^{\circ}$ using SAINT. Analysis of the data showed $0.08 \%$ decay during data collection. Data were corrected for absorption effects with the Semi-empirical from equivalents method using SADABS (Sheldrick, 1996). The minimum and maximum transmission coefficients were 0.933 and 0.991 .

The structure was solved and refined using the SHELXS-97 (Sheldrick, 1990) and SHELXL-97 (Sheldrick, 1997) software in the space group $P 2_{1} / n$ with $Z=2$ for the formula unit $\mathrm{C}_{10} \mathrm{H}_{12} \mathrm{O}_{4}$. The final anisotropic full-matrix least-squares refinement on $\mathrm{F}^{2}$ with 89 variables converged at $\mathrm{R}_{1}=3.54 \%$ for the observed data and $\mathrm{wR}_{2}=7.76 \%$ for all data. The goodness-of-fit was 1.000. The largest peak on the final difference map was $0.262 \overline{\mathrm{e} /} \AA^{3}$ and the largest hole was $-0.181 \overline{\mathrm{e}} / \AA^{3}$. On the basis of the final model, the calculated density was $1.401 \mathrm{~g} / \mathrm{cm}^{3}$ and $\mathrm{F}(000), 208 \overline{\mathrm{e}}$.

Table 1. Sample and crystal data for 4.

\begin{tabular}{|c|c|c|}
\hline X-ray labbook No. & \multicolumn{2}{|l|}{1241} \\
\hline Empirical formula & \multicolumn{2}{|l|}{ C10 H12 O4 } \\
\hline Formula weight & \multicolumn{2}{|l|}{196.20} \\
\hline Temperature & \multicolumn{2}{|l|}{$213(2) \mathrm{K}$} \\
\hline Wavelength & \multicolumn{2}{|l|}{$0.71073 \AA$} \\
\hline Crystal size & \multicolumn{2}{|c|}{$0.45 \times 0.18 \times 0.08 \mathrm{~mm}^{3}$} \\
\hline Crystal habit & \multicolumn{2}{|l|}{ colorless needle } \\
\hline Crystal system & \multicolumn{2}{|l|}{ Monoclinic } \\
\hline Space group & \multicolumn{2}{|l|}{$\mathrm{P} 2{ }_{1} / \mathrm{n}$} \\
\hline \multirow[t]{3}{*}{ Unit cell dimensions } & $\mathrm{a}=8.4475(8) \AA$ & $\alpha=90^{\circ}$ \\
\hline & $\mathrm{b}=5.4646(5) \AA$ & $\beta=99.118(2)^{\circ}$ \\
\hline & $\mathrm{c}=10.2032(9) \AA$ & $\gamma=90^{\circ}$ \\
\hline Volume & $465.05(7) \AA^{3}$ & \\
\hline $\mathrm{Z}$ & 2 & \\
\hline Density, $\rho_{\text {calc }}$ & $1.401 \mathrm{~g} / \mathrm{cm}^{3}$ & \\
\hline Absorption coefficient, $\mu$ & $0.109 \mathrm{~mm}^{-1}$ & \\
\hline $\mathrm{F}(000)$ & $208 \overline{\mathrm{e}}$ & \\
\hline
\end{tabular}


Table 2. Data collection and structure refinement for $\mathbf{4}$.

\begin{tabular}{|c|c|c|}
\hline \multicolumn{2}{|l|}{ Diffractometer } & Bruker Smart1000 CCD area detector \\
\hline \multicolumn{2}{|l|}{ Radiation source } & fine-focus sealed tube, MoK $\alpha$ \\
\hline \multicolumn{2}{|l|}{ Generator power } & $20 \mathrm{kV}, 5 \mathrm{ma}$ \\
\hline \multicolumn{2}{|c|}{ Detector distance } & $4.99 \mathrm{~cm}$ \\
\hline \multicolumn{2}{|c|}{ Detector resolution } & 8.33 pixels $/ \mathrm{mm}$ \\
\hline \multicolumn{2}{|l|}{ Total frames } & 3124 \\
\hline \multicolumn{2}{|l|}{ Frame size } & 512 pixels \\
\hline \multicolumn{2}{|l|}{ Frame width } & $0.3^{\circ}$ \\
\hline \multicolumn{2}{|c|}{ Exposure per frame } & $15 \mathrm{sec}$ \\
\hline \multicolumn{2}{|c|}{ Total measurement time } & 19.2 hours \\
\hline \multicolumn{2}{|c|}{ Data collection method } & $\omega$ and $\varphi$ scans \\
\hline \multicolumn{2}{|c|}{$\theta$ range for data collection } & 2.91 to $27.49^{\circ}$ \\
\hline \multicolumn{2}{|l|}{ Index ranges } & $-10 \leq h \leq 10,-7 \leq k \leq 7,-13 \leq l \leq 13$ \\
\hline \multicolumn{2}{|c|}{ Reflections collected } & 5725 \\
\hline \multicolumn{2}{|c|}{ Independent reflections } & 1058 \\
\hline \multicolumn{2}{|c|}{ Observed reflection, $\mathrm{I}>2 \sigma(\mathrm{I})$} & 962 \\
\hline \multicolumn{2}{|c|}{ Coverage of independent reflections } & $99.9 \%$ \\
\hline \multicolumn{2}{|c|}{ Variation in check reflections } & $0.08 \%$ \\
\hline \multirow{2}{*}{\multicolumn{2}{|c|}{ Absorption correction }} & Semi-empirical from equivalents \\
\hline & & SADABS (Sheldrick, 1996) \\
\hline \multicolumn{2}{|c|}{ Max. and min. transmission } & 0.991 and 0.933 \\
\hline \multicolumn{2}{|c|}{ Structure solution technique } & direct \\
\hline \multicolumn{2}{|c|}{ Structure solution program } & SHELXS-97 (Sheldrick, 1990) \\
\hline \multicolumn{2}{|c|}{ Refinement technique } & Full-matrix least-squares on $\mathrm{F}^{2}$ \\
\hline \multicolumn{2}{|c|}{ Refinement program } & SHELXL-97 (Sheldrick, 1997) \\
\hline \multicolumn{2}{|c|}{ Function minimized } & $\Sigma \mathrm{w}\left(\mathrm{F}_{\mathrm{o}}^{2}-\mathrm{F}_{\mathrm{c}}^{2}\right)^{2}$ \\
\hline \multicolumn{2}{|c|}{ Data / restraints / parameters } & $1058 / 0 / 89$ \\
\hline \multicolumn{2}{|c|}{ Goodness-of-fit on $\mathrm{F}^{2}$} & 1.000 \\
\hline \multicolumn{2}{|l|}{$\Delta / \sigma_{\max }$} & 0.000 \\
\hline \multirow[t]{4}{*}{ Final R indices: } & $\mathrm{R}_{1}, \quad \mathrm{I}>2 \sigma(\mathrm{I})$ & 0.0354 \\
\hline & $w_{2}$, all data & 0.0776 \\
\hline & $\mathrm{R}_{\text {int }}$ & 0.0200 \\
\hline & $\mathrm{R}_{\mathrm{sig}}$ & 0.0131 \\
\hline \multicolumn{2}{|c|}{ Weighting scheme } & $\begin{array}{l}\mathrm{W}=1 /\left[\sigma^{2}\left(\mathrm{~F}_{\mathrm{o}}^{2}\right)+(0.022 \mathrm{P})^{2}+0.2265 \mathrm{P}\right] \\
\mathrm{P}=\left[\max \left(\mathrm{F}_{\mathrm{o}}^{2}, 0\right)+2 \mathrm{~F}_{\mathrm{o}}^{2}\right] / 3\end{array}$ \\
\hline Extinction coeff & ent & $0.018(4)$ \\
\hline Largest diff. pea & and hole & 0.262 and $-0.181 \overline{\mathrm{e}} / \AA^{3}$ \\
\hline
\end{tabular}

$\mathrm{R}_{1}=\Sigma|| \mathrm{F}_{\mathrm{o}}|-| \mathrm{F}_{\mathrm{c}}|/ \Sigma| \mathrm{F}_{\mathrm{o}} \mid, \quad \mathrm{wR} 2=\left[\Sigma \mathrm{w}\left(\mathrm{F}_{\mathrm{o}}^{2}-\mathrm{F}_{\mathrm{c}}^{2}\right)^{2} / \Sigma \mathrm{w}\left(\mathrm{F}_{\mathrm{o}}^{2}\right)^{2}\right]^{1 / 2}$ 
Table 3. Atomic coordinates and equivalent ${ }^{*}$ isotropic atomic displacement parameters $\left(\AA^{2}\right)$ for $\mathbf{4}$.

\begin{tabular}{lllll}
\hline Atom & $x / a$ & $y / b$ & $z / c$ & $\mathrm{U}_{\text {eq }}$ \\
\hline O1 & $0.53240(11)$ & $0.8975(2)$ & $1.15381(9)$ & $0.0471(3)$ \\
O2 & $0.76574(10)$ & $1.04060(16)$ & $1.09670(8)$ & $0.0356(2)$ \\
C1 & $0.65065(14)$ & $0.8700(2)$ & $1.10478(12)$ & $0.0352(3)$ \\
C2 & $0.72900(16)$ & $0.6701(2)$ & $1.03688(15)$ & $0.0386(3)$ \\
C3 & $0.85940(13)$ & $0.8618(2)$ & $1.02584(11)$ & $0.0306(3)$ \\
C4 & $0.87047(14)$ & $0.9548(3)$ & $0.88804(12)$ & $0.0349(3)$ \\
C5 & $0.97791(14)$ & $1.1797(2)$ & $0.89127(13)$ & $0.0360(3)$ \\
\hline
\end{tabular}

${ }^{*} \mathrm{U}_{\mathrm{eq}}$ is defined as one third of the trace of the orthogonalized $\mathrm{U}_{\mathrm{ij}}$ tensor.

Table 4. Anisotropic atomic displacement parameters * $\left(\AA^{2}\right) 4$.

\begin{tabular}{lcccrrr}
\hline Atom & $\mathrm{U}_{11}$ & $\mathrm{U}_{22}$ & $\mathrm{U}_{33}$ & $\mathrm{U}_{23}$ & $\mathrm{U}_{13}$ & $\mathrm{U}_{12}$ \\
\hline $\mathrm{O} 1$ & $0.0355(5)$ & $0.0655(7)$ & $0.0442(5)$ & $0.0051(5)$ & $0.0188(4)$ & $0.0078(4)$ \\
$\mathrm{O} 2$ & $0.0325(4)$ & $0.0373(5)$ & $0.0396(5)$ & $-0.0043(4)$ & $0.0135(3)$ & $0.0040(4)$ \\
$\mathrm{C} 1$ & $0.0306(6)$ & $0.0436(7)$ & $0.0323(6)$ & $0.0043(5)$ & $0.0084(5)$ & $0.0036(5)$ \\
$\mathrm{C} 2$ & $0.0336(6)$ & $0.0374(7)$ & $0.0476(7)$ & $-0.0019(6)$ & $0.0155(5)$ & $-0.0020(5)$ \\
$\mathrm{C} 3$ & $0.0280(6)$ & $0.0310(6)$ & $0.0352(6)$ & $-0.0027(4)$ & $0.0119(4)$ & $0.0030(4)$ \\
$\mathrm{C} 4$ & $0.0277(6)$ & $0.0462(7)$ & $0.0316(6)$ & $-0.0012(5)$ & $0.0072(5)$ & $0.0006(5)$ \\
C5 & $0.0307(6)$ & $0.0414(7)$ & $0.0372(6)$ & $0.0083(5)$ & $0.0097(5)$ & $0.0051(5)$
\end{tabular}

* The anisotropic atomic displacement factor exponent takes the form: $-2 \pi^{2}\left[\mathrm{~h}^{2} \mathrm{a}^{*} \mathrm{U}_{11}+\ldots+2 \mathrm{hka} \mathrm{b}^{*} \mathrm{U}_{12}\right]$

Table 5. Hydrogen atom coordinates and isotropic atomic displacement parameters $\left(\AA^{2}\right)$ for 4 .

\begin{tabular}{ccccc}
\hline Atom & $x / a$ & $y / b$ & $z / c$ & $\mathrm{U}_{\text {iso }}$ \\
\hline $\mathrm{H} 21$ & $0.7624(19)$ & $0.535(3)$ & $1.0941(16)$ & $0.054(5)$ \\
$\mathrm{H} 22$ & $0.6669(18)$ & $0.617(3)$ & $0.9524(16)$ & $0.050(4)$ \\
$\mathrm{H} 41$ & $0.7615(18)$ & $0.991(3)$ & $0.8424(14)$ & $0.041(4)$ \\
$\mathrm{H} 42$ & $0.9163(16)$ & $0.823(3)$ & $0.8404(13)$ & $0.038(4)$ \\
H51 & $0.9259(17)$ & $1.317(3)$ & $0.9293(15)$ & $0.042(4)$ \\
H52 & $0.9922(16)$ & $1.230(3)$ & $0.8014(15)$ & $0.042(4)$ \\
\hline
\end{tabular}

Table 6. Bond lengths $(\AA)$ and angles $\left(^{\circ}\right)$ for 4 .

\begin{tabular}{lllr}
\hline O1-C1 & $1.1954(14)$ & & O2-C1 \\
O2-C3 & $1.5114(13)$ & C1-C2 & $1.3587(15)$ \\
C2-C3 & $1.5374(17)$ & C2-H21 & $1.5025(17)$ \\
C2-H22 & $0.980(16)$ & C3-C4 & $0.954(17)$ \\
C3-C5\#1 & $1.5123(16)$ & C4-C5 & $1.5118(16)$ \\
C4-H41 & $0.983(15)$ & C4-H42 & $1.5251(18)$ \\
C5-H51 & $0.978(15)$ & C5-H52 & $0.983(14)$ \\
& & & $0.982(14)$
\end{tabular}




$\begin{array}{lclr}\text { C1-O2-C3 } & 91.11(8) & \mathrm{O} 1-\mathrm{C} 1-\mathrm{O} 2 & 126.35(12) \\ \text { O1-C1-C2 } & 137.95(13) & \mathrm{O} 2-\mathrm{C} 1-\mathrm{C} 2 & 95.70(9) \\ \text { C1-C2-C3 } & 84.90(9) & \mathrm{C} 1-\mathrm{C} 2-\mathrm{H} 21 & 112.8(10) \\ \text { C3-C2-H21 } & \mathrm{C} 1-\mathrm{C} 2-\mathrm{H} 22 & 114.1(9) \\ \text { C3-C2-H22 } & 115.2(10) & \mathrm{H} 21-\mathrm{C} 2-\mathrm{H} 22 & 111.9(14) \\ \text { O2-C3-C4 } & 115.4(9) & \mathrm{O} 2-\mathrm{C} 3-\mathrm{C} 5 \# 1 & 109.13(9) \\ \text { C4-C3-C5\#1 } & 110.00(9) & \mathrm{O} 2-\mathrm{C} 3-\mathrm{C} 2 & 88.28(8) \\ \text { C4-C3-C2 } & 112.70(9) & \mathrm{C} \text { 5\#1-C3-C2 } & 117.06(11) \\ \text { C3-C4-C5 } & 116.60(11) & \mathrm{C} 3-\mathrm{C} 4-\mathrm{H} 41 & 108.4(8) \\ \text { C5-C4-H41 } & 112.03(10) & \mathrm{C} 3-\mathrm{C} 4-\mathrm{H} 42 & 107.5(8) \\ \text { C5-C4-H42 } & 110.9(9) & \mathrm{H} 41-\mathrm{C} 4-\mathrm{H} 42 & 109.0(12) \\ \text { C3\#1-C5-C4 } & 108.8(8) & \mathrm{C} 3 \# 1-\mathrm{C} 5-\mathrm{H} 51 & 108.6(9) \\ \text { C4-C5-H51 } & 112.09(10) & \mathrm{H} 51-\mathrm{C} 5-\mathrm{H} 52 & 109.2(8) \\ \text { C4-C5-H52 } & 108.8(8) & & 106.7(12) \\ \end{array}$

Symmetry transformation codes: \#1 -x+2,-y+2,-z+2

Table 7. Torsion angles $\left(^{\circ}\right)$ for 4.

\begin{tabular}{lrlr}
\hline C3-O2-C1-O1 & $-179.85(13)$ & C3-O2-C1-C2 & $0.50(10)$ \\
O1-C1-C2-C3 & $179.93(16)$ & O2-C1-C2-C3 & $-0.50(10)$ \\
C1-O2-C3-C4 & $117.28(10)$ & C1-O2-C3-C5\#1 & $-118.61(10)$ \\
C1-O2-C3-C2 & $-0.49(9)$ & C1-C2-C3-O2 & $0.44(9)$ \\
C1-C2-C3-C4 & $-111.13(11)$ & C1-C2-C3-C5\#1 & $111.10(11)$ \\
O2-C3-C4-C5 & $69.53(12)$ & C5\#1-C3-C4-C5 & $-52.49(15)$ \\
C2-C3-C4-C5 & $167.96(10)$ & C3-C4-C5-C3\#1 & $52.16(14)$ \\
\hline
\end{tabular}

Symmetry transformation codes: \#1 -x+2,-y+2,-z+2 